\title{
PSD-95 expression controls L-DOPA dyskinesia through dopamine D1 receptor trafficking
}

\author{
Gregory Porras,, ${ }^{1,2}$ Amandine Berthet,,1,2 Benjamin Dehay, ${ }^{1,2}$ Qin Li, ${ }^{3}$ Laurent Ladepeche,, \\ Elisabeth Normand,4,5 Sandra Dovero,1,2 Audrey Martinez,1,2 Evelyne Doudnikoff,1,2 \\ Marie-Laure Martin-Négrier, ${ }^{1,2}$ Qin Chuan, ${ }^{3}$ Bertrand Bloch, ${ }^{1,2}$ Daniel Choquet,,, 5 \\ Eric Boué-Grabot, ${ }^{1,2}$ Laurent Groc, ${ }^{4,5}$ and Erwan Bezard1,2,3
}

\begin{abstract}
${ }^{1}$ Université de Bordeaux and ${ }^{2}$ CNRS, Institut des Maladies Neurodégénératives, Bordeaux, France. ${ }^{3}$ Institute of Lab Animal Sciences, China Academy of Medical Sciences, Beijing, China. ${ }^{4}$ Université de Bordeaux and ${ }^{5}$ CNRS, Institut Interdisciplinaire des Neurosciences, Bordeaux, France.
\end{abstract}

\begin{abstract}
L-DOPA-induced dyskinesia (LID), a detrimental consequence of dopamine replacement therapy for Parkinson's disease, is associated with an alteration in dopamine D1 receptor (D1R) and glutamate receptor interactions. We hypothesized that the synaptic scaffolding protein PSD-95 plays a pivotal role in this process, as it interacts with D1R, regulates its trafficking and function, and is overexpressed in LID. Here, we demonstrate in rat and macaque models that disrupting the interaction between D1R and PSD-95 in the striatum reduces LID development and severity. Single quantum dot imaging revealed that this benefit was achieved primarily by destabilizing D1R localization, via increased lateral diffusion followed by increased internalization and diminished surface expression. These findings indicate that altering D1R trafficking via synapse-associated scaffolding proteins may be useful in the treatment of dyskinesia in Parkinson's patients.
\end{abstract}

\section{Introduction}

In the striatum, dopamine (DA) terminals from the substantia nigra pars compacta $(\mathrm{SNc})$ converge with glutamatergic signals from the cortex on dendritic spines of striatal medium spiny projecting GABAergic neurons $(1,2)$. The degeneration of the nigrostriatal pathway in Parkinson's disease (PD) induces complex modifications in both DA and glutamate signaling, leading to significant morphological and functional modifications in the striatal neuronal circuitry (3-5). Chronic DA replacement therapy with L-3,4-dihydroxyphenylalanine (L-DOPA) superimposes upon these DA depletion-induced changes, resulting in debilitating motor complications known as L-DOPAinduced dyskinesia (LID) (6-8). At the molecular level, the subcellular organization of and functional interactions between glutamate and DA receptors within the striatum are crucial both in the pathogenesis of PD (9) and in the development of LID $(10,11)$. LID has indeed been associated with plastic changes in postsynaptic neuronal targets in the striatum, including elevated extracellular levels of glutamate (12) and DA (13) and abnormal trafficking of DA D1 receptor (D1R) $(14,15)$ and of NMDA and AMPA glutamate receptor subunits $(5,10,16,17)$. Such exaggerated DA and glutamate receptor expression at the plasma membrane results in abnormal activation of key signaling kinases (18-22). All these changes point to dysfunctional interactions between DA and glutamate neurotransmission in $\operatorname{LID}(5,23,24)$, although the molecular mechanisms remain elusive, despite recent progress $(14,25)$.

The membrane-associated guanylate kinase (MAGUK) proteins, such as postsynaptic density 95 (PSD-95), organize ionotropic glutamate receptors and their associated signaling proteins, regulating the strength of synaptic activity. Interestingly, PSD-95 might also interact with DA D1R (26), thereby potentially regulating DA

Authorship note: Gregory Porras and Amandine Berthet are co-first authors. Laurent Groc and Erwan Bezard are co-senior authors.

Conflict of interest: Erwan Bezard has an equity stake in Motac Holdings Ltd. Erwan Bezard and Qin Li receive consultancy payments from Motac Neuroscience Ltd. Citation for this article: J Clin Invest. 2012;122(11):3977-3989. doi:10.1172/JCI59426.
D1R trafficking and function $(27,28)$. Increased levels of PSD-95 in the synaptic compartment have been reported in a rat model of LID (29), grounding the hypothesis that downregulation of PSD95 levels could decrease the severity of LID by releasing D1R membrane anchorage. In order to test this challenging hypothesis, we here manipulated PSD-95 expression as well as its interaction with $\mathrm{D} 1 \mathrm{R}$ in brain areas of rat and monkey models of dyskinesia and explored its effect at different biological scales, from behavioral outcomes to trafficking of single receptors.

\section{Results}

PSD-95 levels are massively increased in dyskinetic parkinsonian monkeys. Both expression and subcellular distribution of PSD-95 are altered in the striatum of L-DOPA-treated unilateral 6-OHDA-lesioned rats (29), with increased expression and enrichment in the synaptic compartment. To further support our hypothesis, we assessed the status of PSD-95 expression after DA denervation and after DA denervation followed by long-term L-DOPA treatment in the reference experimental model of PD and LID, the 1-methyl-4-phenyl1,2,3,6-tetrahydropyridine-treated (MPTP-treated) macaque monkey $(30,31)$. Immunoblot levels were measured on striatal homogenates collected in normal and MPTP-treated macaque monkeys chronically exposed to vehicle or L-DOPA (see Supplemental Figure 1A for experimental design; supplemental material available online with this article; doi:10.1172/JCI59426DS1). Expression of PSD-95 in monkey putamen was significantly increased in the dyskinetic MPTP-lesioned monkeys compared with the various control animals $(P<0.05$; Figure $1 \mathrm{~A})$. Interestingly, another synapse-associated protein (SAP) - SAP97, reported to be increased in the L-DOPA-treated 6-OHDA rat model of LID (29) - was not increased in the MPTP-lesioned monkeys treated or not with L-DOPA compared with control animals (Supplemental Figure $2 \mathrm{~A})$, which suggests that the PSD-95 increase is specific among SAPs in dyskinesia. To examine whether PSD-95 interacts with DA D1R in the monkey striatum, we performed coimmunoprecipitation experiments on homogenates obtained from monkey striata. 
A
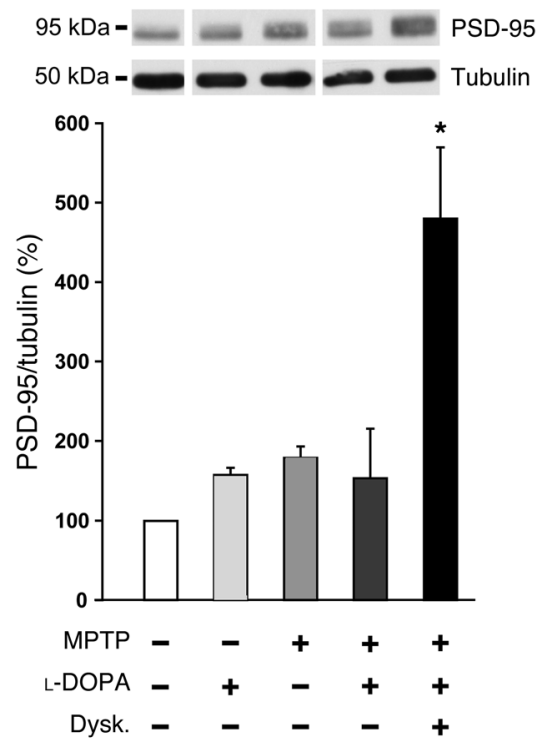

B

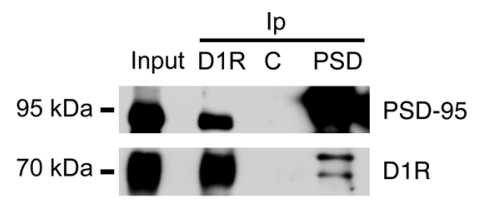

Western blot analysis revealed that anti-D1 antibody immunoprecipitated PSD-95 and, conversely, anti-PSD-95 immunoprecipitated DA D1R (Figure 1B), supporting relevant D1R/PSD complex formation in vivo.

PSD-95 downregulation interferes with behavioral sensitization to L-DOPA in 6-OHDA-lesioned rats. The above findings indicate that increased PSD-95 levels are associated with dyskinesia. We next explored the consequences of overexpressing or downregulating PSD-95 using lentivirus (LV) in the rat analog of dyskinesia, known as the abnormal involuntary movements (AIMs) model (25, 32-34). All $\mathrm{LV}$ transfer vector constructs were modified from the original FUGW vector backbone (35) and encoded GFP (36) carrying PSD95 or shRNA to PSD-95 (referred to as PSD and shPSD groups, respectively; see Methods). Control vectors were a LV encoding GFP (mock group) and a LV encoding for shRNA to PSD-95 plus exogenous shNRA-resistant PSD-95 (SH+PSD group) (36).

We first investigated the effect of regulating PSD-95 expression levels on AIM development (Supplemental Figure 1B). Drug-naive LV-injected 6-OHDA-lesioned rats were treated once daily with L-DOPA, and the frequency of AIMs was scored daily. Repeated administration of L-DOPA to the mock group progressively increased AIM score (Figure 2A and refs. 25, 32). Knockdown of PSD-95 markedly reduced this score compared with the mock group from day 5 onward $(P<0.01$; Figure $2 \mathrm{~A})$, whereas both the PSD and SH+PSD groups were not different from mock rats, suggestive of a ceiling effect for PSD-95. The highlight of AIM time course at day 9 further revealed that AIMs were significantly blunted at all time points following L-DOPA administration in the shPSD versus the mock group $(P<0.05$; Figure $2 \mathrm{~A})$.

PSD-95 downregulation reduces established AIMs in 6-OHDA-lesioned rats. Because antidyskinetic therapy is required for PD patients already suffering from dyskinesia, we tested whether downregulation of PSD-95 would influence preexisting AIMs (Supplemen-

\section{Figure 1}

Increased PSD-95 levels in dyskinetic monkeys and coimmunoprecipitation with D1R. (A) Representative Western blot using anti-PSD-95 and tubulin antibodies from putamen extracts of the different experimental groups (see Supplemental Figure 1). Bar graph represents the relative intensity of the PSD-95/tubulin ratio. Endogenous PSD-95 was significantly increased in the putamen of dyskinetic versus control monkeys. Lanes were run on the same gel but were noncontiguous (white lines). ${ }^{\star} P<0.05$ vs. control. (B) Association between PSD-95 and D1R in monkey striatum. Western blots with anti-PSD-95 or anti-D1 antibodies revealed that PSD-95 and D1R from extracts of dyskinetic monkey striatum tissues coimmunoprecipitated with anti-D1R and anti-PSD antibodies, respectively. No band was detected in absence of antibodies (C, control).

tal Figure 1B). 6-OHDA-lesioned rats were treated with L-DOPA for 9 days, and AIM scores were found to be fully comparable among groups, all of which displayed severe $\operatorname{AIMs}(P>0.5$; Figure 2B). LVs were stereotactically injected on day 12 (Figure 2B), and L-DOPA treatment was resumed on day 22. Peak AIM score in the shPSD group was significantly reduced compared with the mock group from day 24 onward $(P<0.01$; Figure $2 \mathrm{~B})$, whereas both the PSD and SH+PSD groups were not different from mock rats. The highlight of AIM time course at day 27 further revealed that AIMs were significantly decreased for the whole time course after L-DOPA administration in the shPSD versus the mock group $(P<0.001 ;$ Figure 2B). Interestingly, shPSD did not modify SAP97 levels (Supplemental Figure 2B), which suggests that the shPSDmediated behavioral effect does not involve compensation for reduced PSD-95 levels by increasing expression of this other SAP. These behavioral data were further supported by the significant decrease in pERK immunoblot levels, a signaling marker classically associated with AIMs $(20,21,37)$, in the shPSD versus the mock group (pERK/total ERK; mock, $125.9 \pm 2.9$ AU; shPSD, $104.0 \pm 8.8$ AU; $P<0.05$, unpaired $t$ test). Similarly, the mRNA level of cytochrome oxidase subunit I (COX I; a marker whose expression correlates with electrical activity; refs. 38, 39), as determined by qPCR in the substantia nigra pars reticulata $\mathrm{SNr}$; the output structure of the basal ganglia) was higher in the shPSD than in the mock group ( $21 \%$ increase; $P=0.032$ ). This suggests that improvement in behavior correlates with normalization of the overdecreased $\mathrm{SNr}$ activity in dyskinetic animals $(40,41)$.

We examined the expression level of endogenous PSD-95 as well as exogenous PSD-95-GFP by Western blotting using anti-PSD95 antibodies in rat striatum of animals infected with the different LVs. Detection of 2 bands ( 95 and $125 \mathrm{kDa}$ ) corresponding to endogenous and exogenous GFP-tagged PSD-95 in animals infected with PSD-95-GFP alone or shPSD+PSD-95-GFP (Figure $2 \mathrm{C}$ ) was indicative of the efficiency of LV-driven expression in both set of experiments. Importantly, the expression of endogenous PSD-95 was significantly diminished in presence of shPSD. Endogenous PSD-95 represented about $45 \%(P<0.01)$ and $65 \%$ $(P<0.05)$ of the control mock group in experiments 1 and 2 , respectively (Figure 2, A and B). Biochemical demonstration of PSD-95 downregulation was further confirmed in the striatum by double immunohistochemistry for GFP and PSD-95 (Supplemental Figure 3). Comparable large areas of the dorsal motor striatum were GFP-immunopositive (Supplemental Figure 3A) across the 4 groups, with medium spiny-like neurons being clearly labeled (Supplemental Figure 3B). The shPSD group displayed a severe 


\section{A}
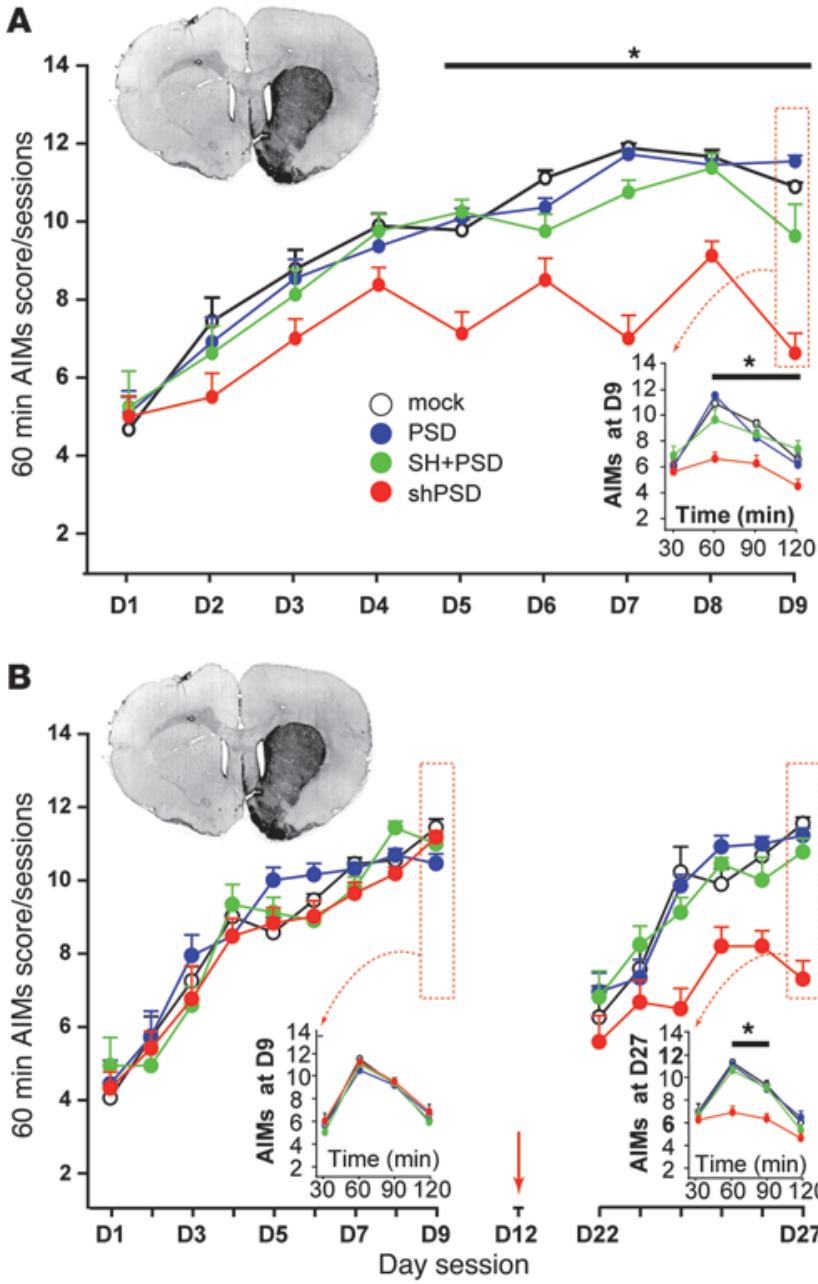

C

L UL L UL L UL L UL
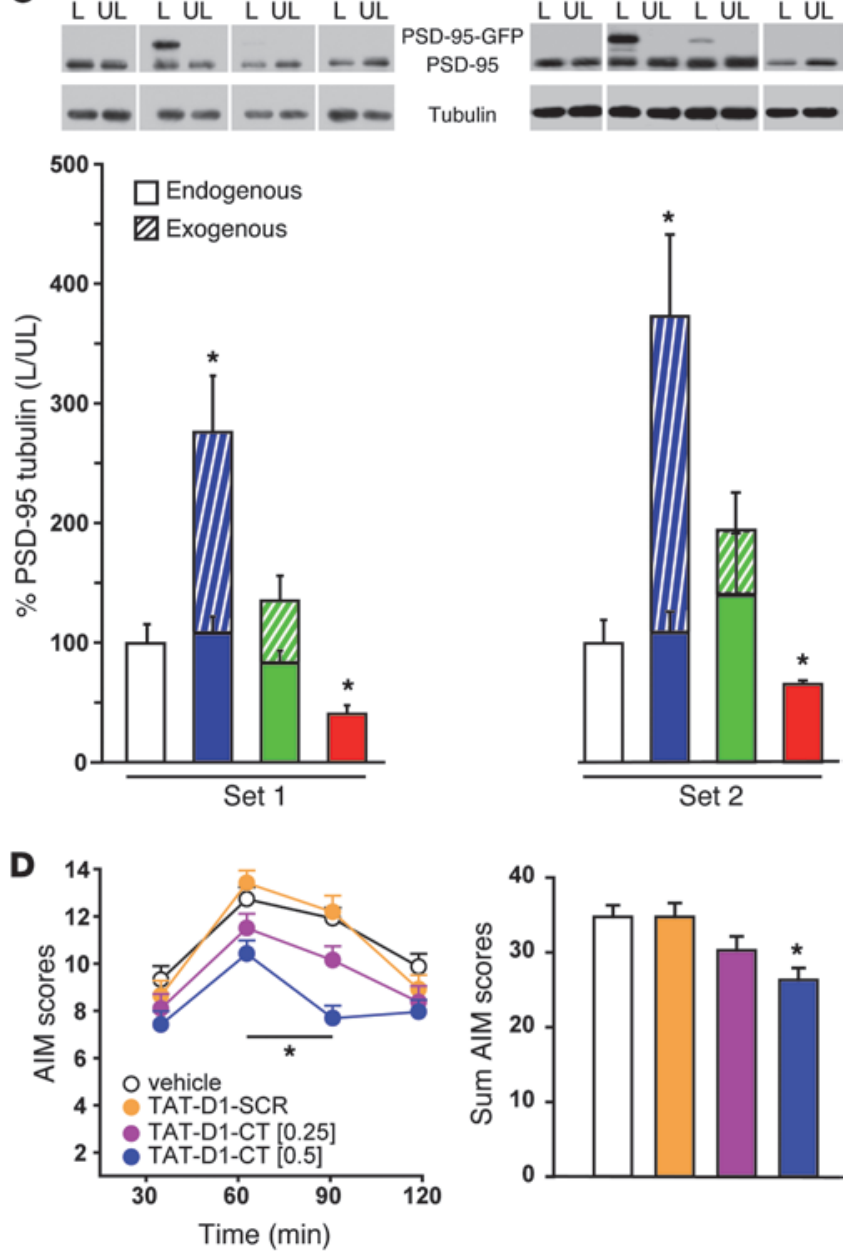
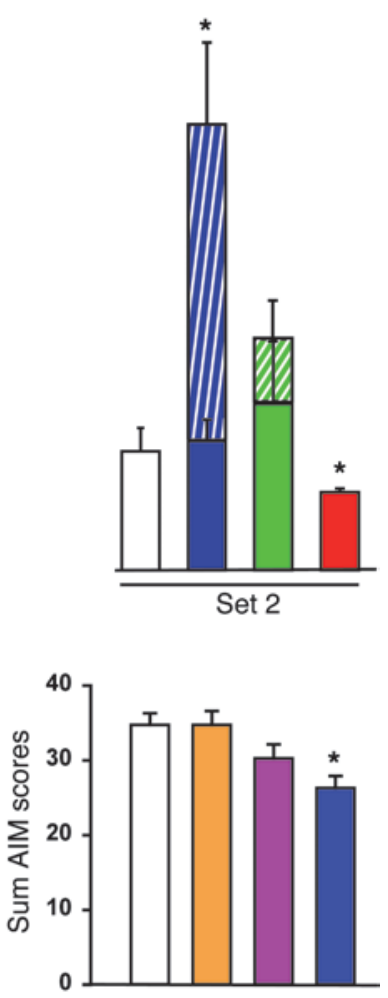

Figure 2

Downregulating PSD-95 in the DA-deprived striatum reduces AIM severity in the hemiparkinsonian rat. (A) Both the peak AIM scores (rated at 60 minutes) from day 5 as well as the time course AIM (bottom right inset) scores were significantly reduced with shPSD LV compared with mock control. ${ }^{\star} P<0.05$, shPSD vs. mock. (B) Rats displayed identical peak AIMs after 9 L-DOPA treatment days before receiving LV at day 12 (red arrow). Peak AIM score in the shPSD group was significantly reduced compared with all other groups. This reduction was further highlighted in a time course experiment at day 27 (bottom right insets), with a significant decrease 60 and 90 minutes after L-DOPA administration. In both sets, animals had similar loss of dopaminergic innervation in the striatum as detected by $\mathrm{TH}$ immunohistochemistry (top left insets). ${ }^{*} P<0.05$, shPSD vs. all other groups. (C) Detection of the endogenous PSD-95 and exogenous PSD-95-GFP transgene by Western blot in striata extracts from 2 sets of animals. Both lesioned (L) and unlesioned (UL) sides are shown. In both experiments, shPSD significantly reduced the expression of endogenous PSD-95, whereas PSD significantly increased PSD-95, compared with the mock group. Lanes were run on the same gel but were noncontiguous (white lines). ${ }^{*} P<0.05$ vs. mock. (D) While TAT-D1-SCR peptide had no effect on AIM score, TAT-D1-CT (0.25 and $\left.0.5 \mathrm{nmol}\right)$ dose-dependently reduced AIM score at peak and at 60 and 90 minutes after L-DOPA administration, resulting in overall improvement. ${ }^{*} P<0.05$ vs. vehicle.

reduction of PSD-95 immunoreactivity, with most medium spinylike neurons being immunopositive only for GFP (Supplemental Figure 3B). 3-dimensional reconstruction of LV-transfected rat brain showed that the striatum was largely transfected $(>50 \%)$ in the 4 groups (Supplemental Movie 1). Together, these controls demonstrated the efficiency of the targeting and of the reduction in PSD-95 levels in the shPSD group, a result compatible with the marked reduction in AIM severity in these animals.

Cell-permeable peptide disrupting the PSD-95-D1R interaction reduces established AIMs in 6-OHDA-lesioned rats. An alternate option to lowering PSD-95 levels for preventing the interaction of PSD-95 with $\mathrm{D} 1 \mathrm{R}$ is to design (and administrate) competitive peptides corre- sponding to the domain of rat D1R interacting with PSD-95 (42) that would disrupt D1R/PSD-95 interaction. We first examined the ability of the distal region of the C-terminal tail of D1R to bind to PSD-95 by performing pulldown assays with active (D1-CT) and control (D1-SCR) peptides (Supplemental Figure 4). Extracts of monkey or rat brain tissues as well as HEK293 cells expressing PSD95 or PSD-95-GFP were incubated in the presence of immobilized biotinylated peptides. PSD-95 coprecipitated with the active D1-CT peptide (Supplemental Figure 4), which indicates that D1-CT interacts with native or recombinant PSD-95 from different species. In addition, detection of PSD-95-GFP indicated that fusion of the GFP does not alter function of PSD-95 related to D1R. 
A

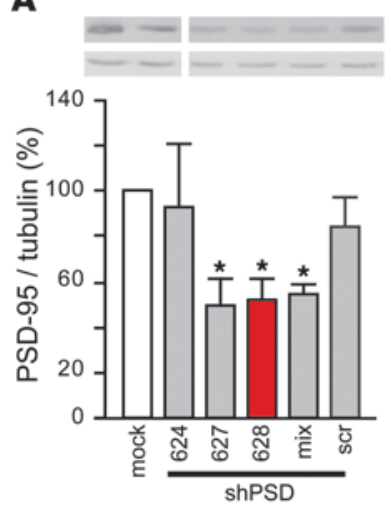

C

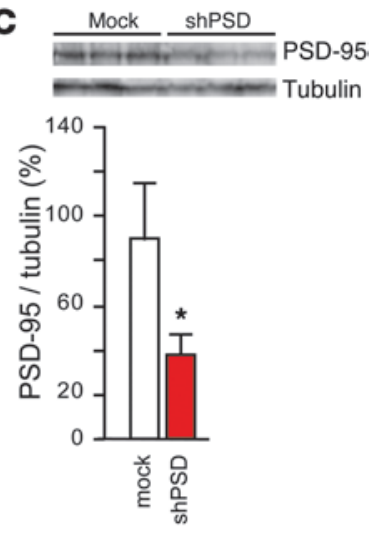

B

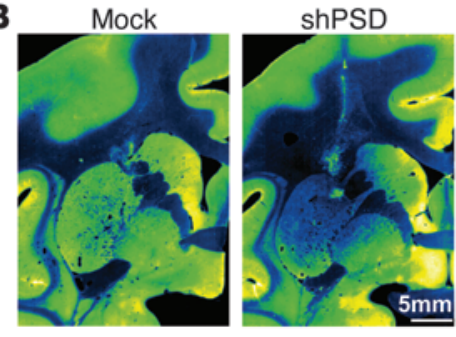

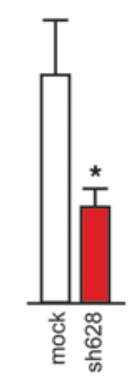

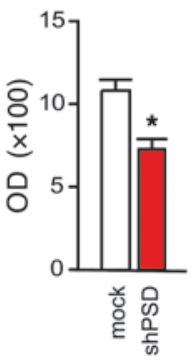

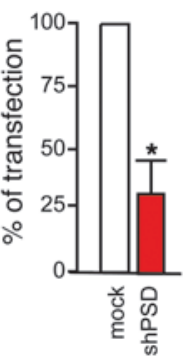

\section{Figure 3}

shPSD LV reduces PSD-95 levels in the MPTPtreated macaque. (A) 3 human shRNA sequences (sh624, sh627, and sh628) were tested separately or together (mix) in neuroblastoma human cells. sh627, sh628, and the mix of the 3 shRNAs displayed a significant reduction in PSD-95 levels compared with the mock group. Integration of the sh628 sequence into a LV induced in the same cell line significantly decreased the PSD-95 level. Lanes were run on the same gel but were noncontiguous (white lines). ${ }^{\star} P<0.05$ vs. mock. (B) Representative photomicrograph of motor striatal sections infected with mock or ShPSD vector. Brain sections were immunostained with an antiPSD-95 antibody (green) to label endogenous PSD-95, and fluorescence was visualized by confocal microscopy. Quantitative staining analysis confirmed that PSD-95 expression was significantly reduced in the shPSD group, affecting most of the motor postcommissural putamen. Scale bar: $5 \mathrm{~mm}$. ${ }^{*} P<0.05$ vs. mock. (C) PSD-95 Western blot run on striata scrapped from sections close to injection site further showed that shPSD LV significantly decreased PSD-95 level. ${ }^{*} P<0.05$ vs. mock.
Both peptides were fused to the cell membrane transduction domain of the human immunodeficiency virus 1 (i.e., the TAT signal) to gain cell permeability (herein, we refer to the control as TAT-D1-SCR and the active form as TAT-D1-CT). We tested whether administration of TAT-D1-CT would reduce the severity of established AIMs (Supplemental Figure 1B), as in the shPSD experiment. 6-OHDA-lesioned rats were treated with L-DOPA for 21 days. TAT peptides were injected through intrastriatal cannulae at the same time as L-DOPA administration. While TAT-D1-SCR did not affect AIM score (Figure 2D), TATD1-CT dose-dependently reduced AIM scores $(P<0.05$; Figure $2 \mathrm{D})$. The AIM time course showed that AIMs were significantly decreased at peak dyskinesia, i.e., at 60 and 90 minutes $(P<0.05)$ after L-DOPA administration, in the highest dose of the TATD1/PSD condition.

Decreasing PSD-95 levels alleviates LID in MPTP-lesioned macaques. The rat experiments clearly suggested that reducing PSD-95 expression or competing with D1R/PSD-95 interaction interferes with the development (Figure 2A) and reduces the severity (Figure 2, B-D) of AIMs. When drug treatment was resumed on day 22, however, animal LID had decreased $40 \%-50 \%$. At the end of day 27 , all but the shPSD group was back to the same level of LID as on day 9, while shPSD LID remained low. However, since LID in the 6-OHDA rat model might not have fully consolidated, final preclinical proof-of-concept evidence for an antidyskinetic strategy should be obtained in the fully stable MPTP-lesioned macaque model of PD, as clinical manifestations resemble the severe peak dose LID in human PD $(30,31)$. We therefore investigated the effectiveness of the shPSD strategy in the stably dyskinetic MPTPlesioned macaque monkey as well as the preservation of L-DOPAmediated antiparkinsonian action.

First, we selected the shRNA plasmids against human PSD-95 with the maximum efficiency of silencing endogenous PSD-95 in the BE-M17 human cell line (Figure 3A). We concluded that sh628 was the best candidate, with a $50 \%$ decrease in expression $(P<0.05)$. The ability of the $\mathrm{LV}$ encoding sh 628 to silence human PSD-95 was tested in the same human cell line (Figure 3A).

MPTP-treated macaques rendered dyskinetic by chronic L-DOPA treatment (Supplemental Figure 1A) received mock (encoding GFP; $n=3)$ or $\operatorname{shPSD}(n=4) \mathrm{LV}$ in the motor striatum. Upon completion of the behavioral experiments, all monkeys were tested to evaluate the extent of lesion and transduction efficacy. Both groups had similarly extensive nigrostriatal cell loss, as evidenced by the dramatic decrease in the number of tyrosine hydroxylase-immunopositive ( $\mathrm{TH}$-immunopositive) neurons in the SNc (unbiased stereological counting; mock, 13,700 $\pm 2,729$; shPSD, $12,800 \pm 1,658$; unlesioned controls, $126,300 \pm 3,241)$. PSD-95 immunohistochemistry revealed a significant decrease in PSD-95 levels of the shPSD versus the control group (Figure 3B), a decrease that covered $67.9 \% \pm$ $12.8 \%$ of the postcommissural motor putamen (Figure 3B). Confirmation of the decrease in endogenous PSD-95 after ShPSD LV was obtained by Western blot of homogenized striatal tissues collected on sections in the motor striatum (Figure 3C).

Prior to LV administration, parkinsonian disability scores in both the Off and On states (before and after L-DOPA administration, respectively; Figure 4A), LID scores in the On state (Figure 4C), and the time course of L-DOPA-induced locomotor activity (Figure 4E) were comparable. Starting at 4 weeks postsurgery, when behavioral experiments resumed, PD and LID scores as well as locomotor activity counts in the mock group were fully comparable to those prior to surgery (Figure 4, B, D, and F). In test animals, the antiparkinsonian efficacy of L-DOPA remained intact in mock versus shPSD animals (Figure 4, A and B). However, shPSD monkeys had significantly less severe LID (Figure 4, C and D) and a significant decrease in L-DOPA-induced locomotor hyperactivity in the On state (Figure 4, E and F). Since locomotor activity counts capture both the antiparkinsonian and prodyskinetic actions of L-DOPA, an antidyskinetic strategy would reduce 
A
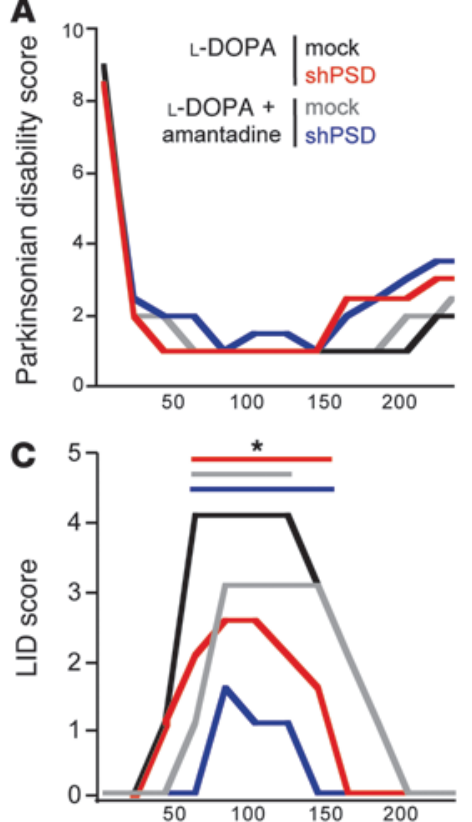

E
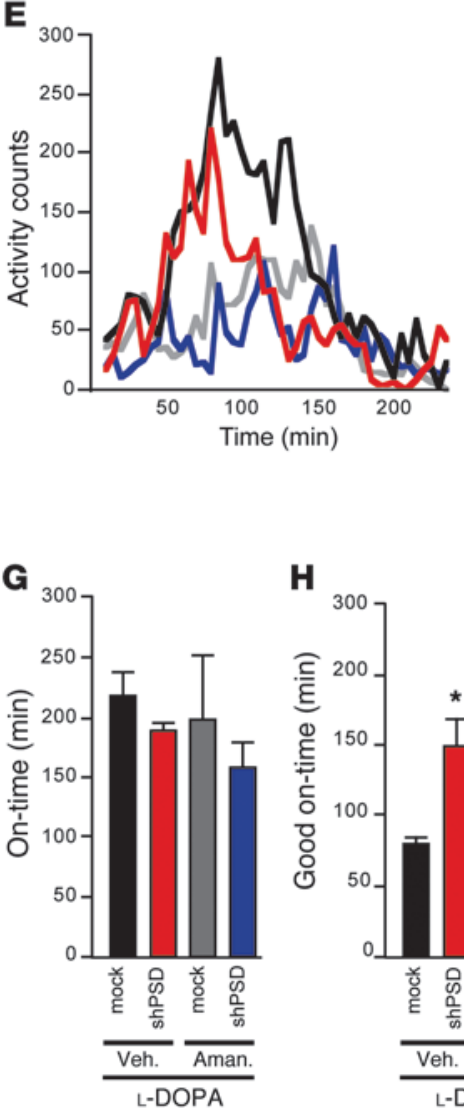

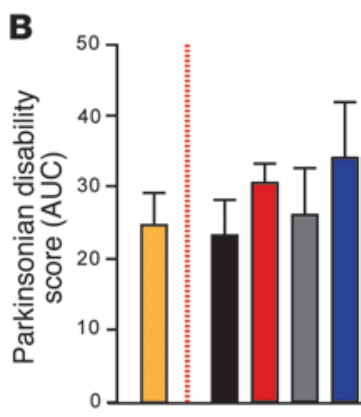

D

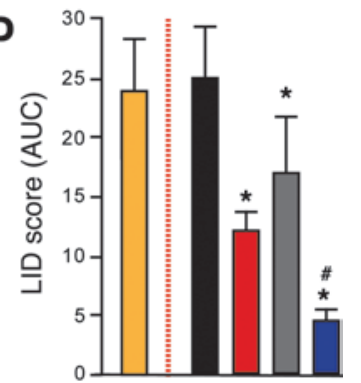

$\mathbf{F}$

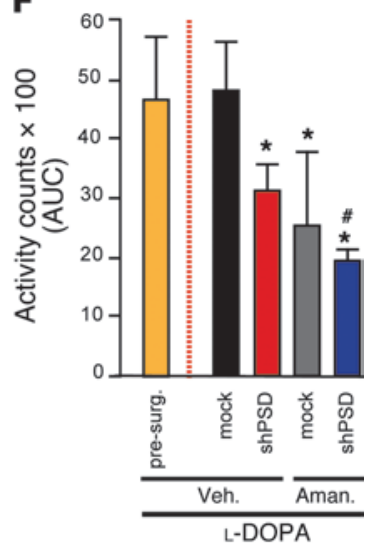

I

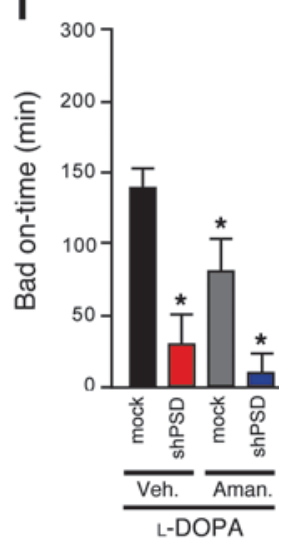

the activity counts, reflecting the reduction of hyperkinetic movements, but would not suppress them, or it would indicate an antiL-DOPA effect. Measurement of On-time also showed that shPSD animals were comparable to the mock group (Figure 4G). In fact, while On-time per se was not affected, shPSD animals benefited from shPSD LV, with a significant increase in Good On-time (Figure $4 \mathrm{H}$ ) and a concomitant significant decrease in Bad On-time

\section{Figure 4}

Reducing PSD-95 in the MPTP-treated macaque markedly alleviates dyskinesia. (A, C, and E) Time course of clinical ratings (median) and locomotor activity counts (mean). Data are shown without SEM for readability. L-DOPA or amantadine administration began at 0 minutes. (A) shPSD had no effect on L-DOPA-induced parkinsonian disability score at any time point. (B) Area under the curve (AUC) analysis of parkinsonian disability scores confirmed this lack of effect. Orange bar denotes the presurgery score of the entire population. (C) LID severity was affected by shPSD and/or pharmacological treatment. ${ }^{*} P<0.05$ vs. L-DOPA-treated mock (bracket colors correspond with respective groups to show time frame of significance). (D) The overall positive effect of shPSD and/or pharmacological treatment on LID severity was further demonstrated by the AUC data. ${ }^{*} P<0.05$ vs. L-DOPA-treated mock; ${ }^{~} P<0.05$ vs. L-DOPA-treated shPSD. Orange bar denotes the presurgery score of the entire population. (E and $\mathbf{F}$ ) Consequently, locomotor activity was lower in ShPSD animals receiving L-DOPA alone and in both groups when receiving L-DOPA plus amantadine. Orange bar denotes the presurgery count for the entire population. ${ }^{*} P<0.05$ vs. L-DOPA-treated mock; ${ }^{P}<0.05$ vs. L-DOPA-treated shPSD. (G) On-time was not affected by any treatment. (H) shPSD significantly increased Good On-time. ${ }^{*} P<0.05$ vs. L-DOPA-treated mock. (I) Bad On-time was reduced in ShPSD animals receiving L-DOPA alone and in both groups when receiving L-DOPA plus amantadine. ${ }^{*} P<0.05$ vs. L-DOPA-treated mock.

(Figure 4I). These are key translational parameters, as they quantitatively reflect the possible qualitative improvement to be reported by dyskinetic patients enrolled in a clinical trial. The combination of the clinically assessed decrease in LID scores, reduced locomotor activity, and stable On-time with a marked increase in Good On-time supports our hypothesis that striatal downregulation of PSD-95 diminishes LID severity without reducing the positive effects of L-DOPA on parkinsonian motor scores.

Although the LID-related rise in PSD-95 levels (Figure 1A) was only reduced $40 \%$ by the shPSD LV (Figure 3B), i.e., PSD-95 levels were not brought back to normal levels, one cannot rule out a possible impairment of the glutamate corticostriatal transmission. At present, amantadine, an antagonist at the N-methyl-D-aspartate (NMDA) subtype of glutamate receptor, is the drug most often used for the treatment of LID (43). Mock animals displayed the expected reduction in LID severity after acute pharmacological challenge with amantadine $(20 \mathrm{mg} / \mathrm{kg}$ orally; ref. 44$)$ in combination with L-DOPA (Figure 4, C and D) without reduction in antiparkinsonian response to L-DOPA, as evidenced by parkinsonian disability score (Figure 4, A and B), locomotor activity profile (Figure 4, E and F), and On-time (Figure 4G). However, shPSD animals further benefited from amantadine treatment: LID severity of shPSD animals was significantly different not only from mock animals treated with L-DOPA only, but also from shPSD animals treated with L-DOPA only (Figure 4, C and D) Such additive effect was also significant for the locomotor activity counts (Figure 4, E and F). The combination of L-DOPA and amantadine in shPSD animals resulted into an almost complete suppression of Bad On-time (Figure 4I). Together, these data suggest that NMDA-mediated corticostriatal transmission is not blunted in shPSD animals, as they additively benefited from combination L-DOPA and amantadine therapy.

PSD-95 downregulation alters D1R surface content and trafficking. The binding of D1R and glutamate receptors to the scaffold protein PSD-95 $(28,42)$ has provided a molecular mechanism by which these receptors are anchored in synaptic areas and by which these 2 
A
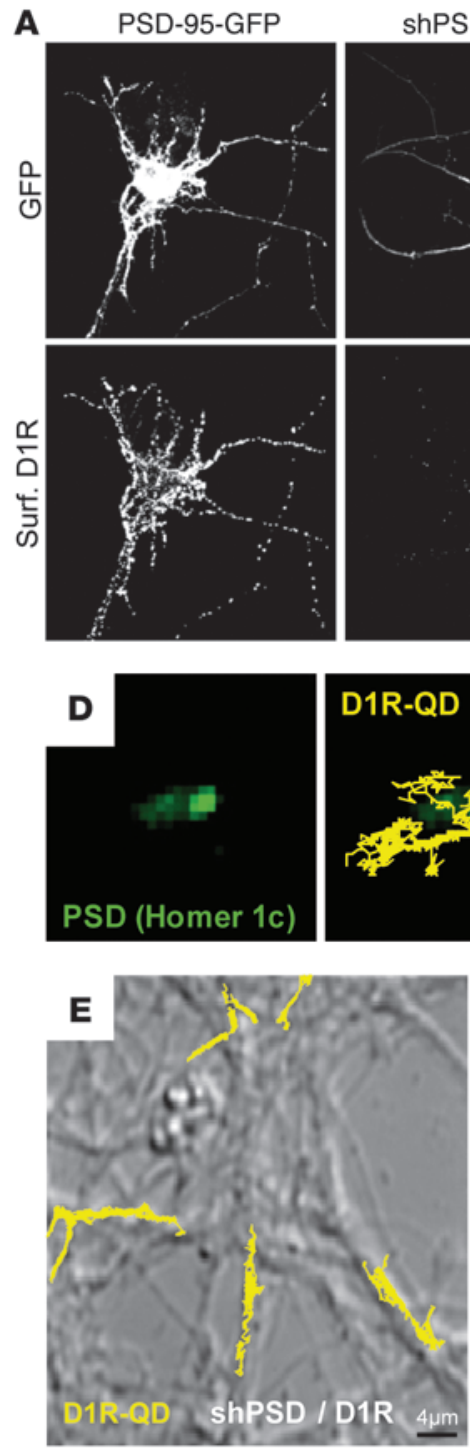

$\mathbf{F}$

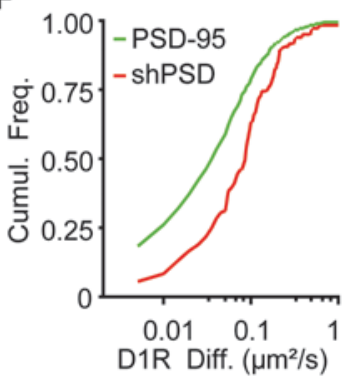

B

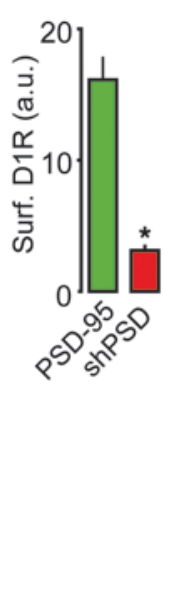

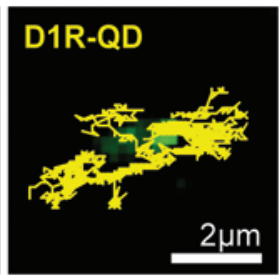

$2 \mu \mathrm{m}$
C

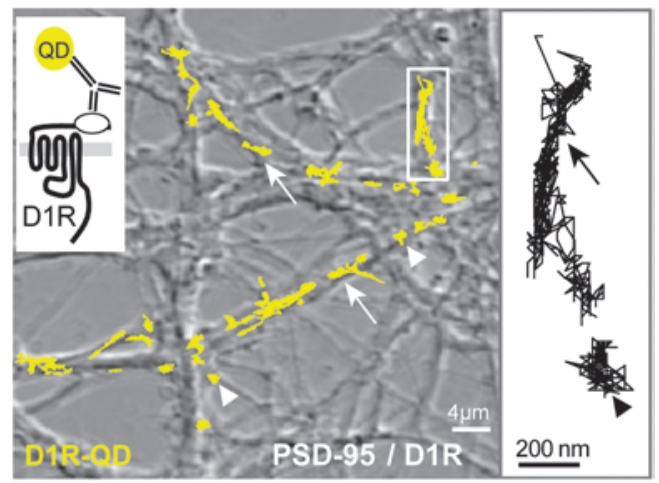

I TAT-D1-SCR

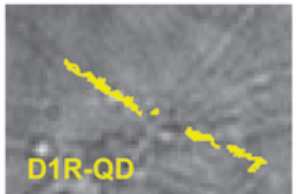

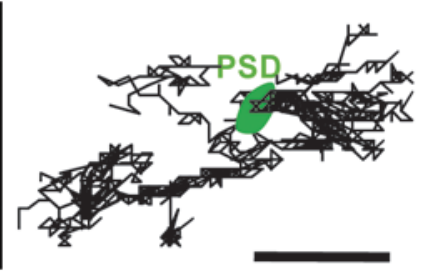

G

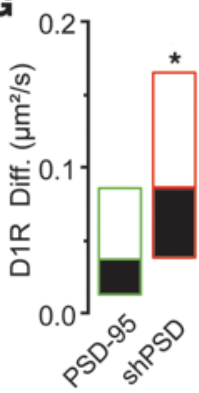

H

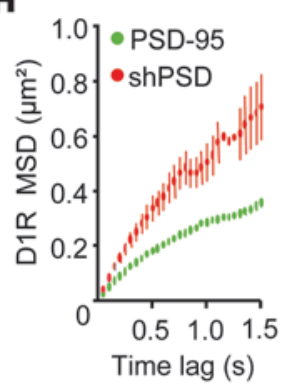

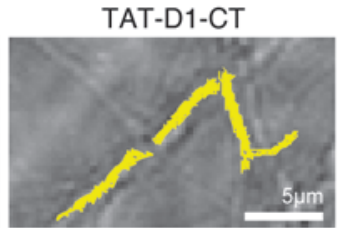

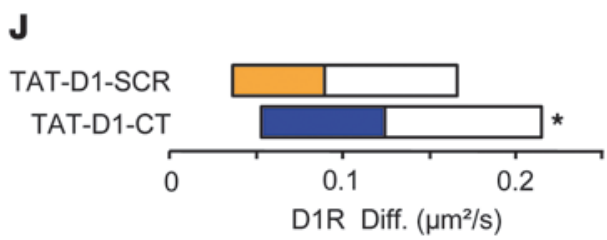

\section{Figure 5}

PSD-95 knockdown or peptide disrupting PSD-95-D1R interaction alters D1R surface content and dynamics on cultured striatal neurons. (A and B) Immunostaining of surface D1R (A) showed a significant decrease in surface D1R in shPSD neurons (B). Scale bar: $16 \mu \mathrm{m}$. ${ }^{\star} P<0.05$. (C) Representative trajectories of surface D1Rs labeled with a single QD-antibody complex (inset) at the surface of a PSD-95expressing neuron. A fraction rapidly diffused along dendrites and explored large areas (arrows), whereas others diffused in a confined area (arrowheads). Scale bars: $4 \mu \mathrm{m}$ (left); $200 \mathrm{~nm}$ (right). (D) Representative trajectories of surface D1R on a Homer1c-GFP-expressing neuron. Also shown are distributions (median and $25 \%-75 \%$ IQR) of the instantaneous diffusion coefficient of surface D1Rs inside and outside the PSD area. Scale bar: $2 \mu \mathrm{m}$. ${ }^{*} P<0.05$. (E) Representative trajectories of surface D1Rs on a shPSD-expressing striatal neuron. Scale bar: $4 \mu \mathrm{m}$. (F) Cumulative distribution of the instantaneous diffusion coefficient of surface D1Rs, starting from the percentage of immobile receptors. (G) Corresponding distributions of the instantaneous diffusion coefficient of surface D1Rs. ${ }^{*} P<0.05$. (H) The relationship between mean square displacement (MSD) and time of surface D1R trajectories was not linear (dotted line denotes free diffusion), adopting negative curvature, characteristic of confined behavior (Kolmogorov-Smirnov test). (I and $\mathbf{J}$ ) Representative trajectories (I) and distributions of the instantaneous diffusion coefficient $(\mathbf{J})$ of surface D1Rs in the presence of TAT-D1-SCR or TAT-D1-CT. Scale bar: $5 \mu \mathrm{m}$. ${ }^{*} P<0.05$. 
different pathways may communicate $(27,45)$. The above data provide clear evidence that PSD-95 expression affects the emergence of LID, which is greatly dependent on DA receptor-mediated signaling $(30,31)$. Because postmortem assessment of DA, metabotropic, and ionotropic glutamate receptors in the rat striatum did not show any dramatic regulation of the receptor content after over- or downregulation of PSD-95 (Supplemental Figure 5), we decided to identify, at the molecular level, the relationship between PSD-95 and surface D1R signaling using a combination of single nanoparticle tracking and immunocytochemical approaches in cultured striatal neurons. In this in vitro preparation, PSD-95 knockdown (i.e., shPSD) significantly reduced the cellular content of PSD-95 by approximately 30\% (Supplemental Figure 6), a value equivalent to those observed in rat and monkey striata (Figure $2 \mathrm{C}$ and Figure 3, B and C). We then investigated the effect of PSD-95 knockdown on the cellular distribution of D1R. Consistent with the effect observed in the rat brain, the global content of D1R was not significantly altered in shPSD-transfected neurons compared with neighboring untransfected ones (Supplemental Figure 6). However, the surface content of D1R was drastically reduced (4-fold decrease) in the shPSD condition (Figure 5, A and $\mathrm{B})$, in both the dendritic and the somatic compartments. Because PSD-95 strongly interacts with NMDAR and anchors the receptor to the synapse $(46,47)$, we investigated the effect of PSD-95 knockdown on the surface expression of GluN2B-NMDAR (Supplemental Figure 6). In contrast to the effect observed with D1R, the surface content was similar in shPSD-transfected and neighboring untransfected neurons (Supplemental Figure 6), which indicates that PSD-95 altered the surface content of D1R, but not of NMDAR, in striatal neurons. Whether the surface dynamics of different NMDAR subtypes are affected in these conditions, however, remains to be explored. Together, these data demonstrate that PSD-95 knockdown affects D1R surface content without any significant effect on its global one, suggestive of specific modulation of receptor surface trafficking.

To further identify the pathways involved in PSD-95-dependent regulation of D1R trafficking, we used a single-particle tracking approach (quantum dot [QD]; ref. 48) to follow over time single D1R-QDs at the surface of striatal neurons (Figure 5C). A small fraction of surface D1R-CFP was labeled with anti-GFP antibody coupled to a single QD in live striatal neurons. In PSD-95-expressing neurons, surface D1Rs were highly mobile and diffusive (Figure 5C). Representative trajectories from a 60-second imaging session revealed that most D1Rs diffused (median instantaneous diffusion coefficient, $0.04 \mu \mathrm{m}^{2} / \mathrm{s}$; interquartile range [IQR], $0.01-0.08 \mu \mathrm{m}^{2} / \mathrm{s} ; n=2,695$ trajectories), although a confined pattern of diffusion was observed (Figure 5C). Because PSD-95 itself can affect the surface dynamics of D1R, we measured the surface diffusion of D1R in neurons expressing Homer1c, another protein of the postsynaptic density that does not interact with D1R. The diffusion of D1R in these neurons was not significantly different from the one in neurons expressing PSD-95 (median instantaneous diffusion coefficient, $0.03 \mu \mathrm{m}^{2} / \mathrm{s}$; IQR, 0.001-0.07 $\mu \mathrm{m}^{2} / \mathrm{s}$; $n=312$ synaptic and 1,695 extrasynaptic trajectories; Figure 5D). We took advantage of the presence of Homer1c clusters, indicative of postsynaptic density, to investigate D1R diffusion characteristics in glutamate synapses. D1Rs were transitorily retained in the PSD area. D1Rs were thus less diffusive in the vicinity and within PSD (Figure 5D), consistent with the presence of anchoring mechanisms for surface D1R in glutamate synapses.
In the basal condition, more than $80 \%$ of surface D1Rs were mobile at the surface of striatal neurons (expressing wild-type PSD-95 or Homer1c). In contrast, PSD-95 knockdown produced a substantial change in D1R surface trafficking (800-frame duration; Figure 5E). Indeed, the overall surface diffusion of D1Rs was significantly increased, with more than $94 \%$ mobile receptors (bin size, $0.005 \mu \mathrm{m}^{2} / \mathrm{s} ; n=121-2,695$ trajectories; Figure $5 \mathrm{~F}$ ). The $\mathrm{D} 1 \mathrm{R}$ median instantaneous diffusion coefficient was significantly increased, from 0.04 to $0.09 \mu \mathrm{m}^{2} / \mathrm{s}$ (IQR, 0.04-0.16 $\mu \mathrm{m}^{2} / \mathrm{s}$ ), in PSD-95 and shPSD conditions, respectively $(n=121-2,695$ trajectories; Figure 5G). In addition, the D1R diffusion pattern was examined by plotting the mean squared displacement (surface explored by the receptor) versus time interval (49). In PSD-95expressing neurons, the D1R surface diffusion pattern was not purely Brownian (comparison of 56 trajectories per group; Figure $5 \mathrm{H}$ ), suggestive of the presence of regulatory mechanisms at the neuronal surface. In shPSD conditions, the diffusion pattern abruptly changed toward free diffusion (Figure $5 \mathrm{H}$ ), which indicates that the surface trafficking of D1R was highly regulated by the presence of PSD-95. To investigate the role of the direct interaction between D1R and PSD-95, we incubated neurons with the TAT-D1-CT peptide, which disrupted interaction between D1R and PSD-95, and measured its acute effect on D1R surface trafficking. TAT-D1-CT ( $5 \mu \mathrm{M}$ for 10 minutes) produced an increase diffusion of surface D1R (Figure 5I). The D1R median instantaneous diffusion coefficient was significantly increased, from 0.08 $\mu \mathrm{m}^{2} / \mathrm{s}$ for TAT-D1-SCR (IQR, 0.03-0.16 $\mu \mathrm{m}^{2} / \mathrm{s} ; n=2,619$ trajectories) to $0.125 \mu \mathrm{m}^{2} / \mathrm{s}$ for TAT-D1-CT (IQR, $0.05-0.215 \mu \mathrm{m}^{2} / \mathrm{s}$; $n=3,924$ trajectories; $P<0.01$ between groups; Figure 5J). Since the peptide described may interfere with the interaction between GluN2A and D1R, we confirmed that TAT-D1-CT (5 $\mu \mathrm{M}, 10$ minutes) did not alter the content or distribution of GluN2A or GluN2B subunits (Supplemental Figure 7). Together, these data indicated that PSD-95 plays an important role in the surface content and dynamics of D1R in striatal neurons.

\section{Discussion}

We demonstrated here that disrupting D1R and PSD-95 interaction in the striatum of dyskinetic rats and monkeys, through either lowering PSD-95 levels or blocking direct interaction using competing peptides, reduced dyskinesia severity. This outcome was achieved by destabilizing D1R localization through increased lateral diffusion, possibly leading to increased internalization and thus less surface expression.

PSD-95 is at the crossroad of glutamate and DA signal modulation (47), especially as glutamatergic and DA axons form a synaptic triad at postsynaptic dendritic spines in the striatum, a close spatial association that is required for the converging and coordinating actions of the 2 afferent pathways (50). LID is associated with abnormal trafficking of DA D1Rs $(14,15)$ and of NMDA and AMPA glutamate receptor subunits $(5,10,16,17)$. Such exaggerated DA and glutamate receptor expression at the plasma membrane results in abnormal activation of key signaling kinases $(18-22,37)$. The molecular mechanisms mediating such lack of trafficking that causes enhanced anchorage and function remain the subject of debate. Alteration of the DA D1R homologous desensitization process is partly responsible for such anchoring $(14,51)$, as increasing the capacity of the desensitization machinery $(52,53)$ in the parkinsonian striatum ameliorates $\operatorname{LID}(14,25)$. In the same way, the redistribution of NMDA gluta- 
mate receptor subunits with higher levels of GluN2A and lower levels of GluN2B $(16,54)$ is paralleled by profound modifications of NMDA receptor subunit association with interacting elements (47), i.e., MAGUK protein family members PSD-95 and SAP (29, 54). Treatment of nondyskinetic animals with TAT2B, a synthetic peptide able to affect GluN2B binding to MAGUK proteins as well as synaptic localization of this subunit in nondyskinetic rats, was sufficient to induce a shift of treated rats toward dyskinetic motor behavior (54). Comparably, another peptide directly targeting PSD-95-GluN2A subunit interactions, TAT2A, could reverse motor and synaptic plasticity abnormalities in early parkinsonism (55). These data indicate that interaction of NMDA subunits with PSD-95 governs their redistribution between synaptic and extrasynaptic membranes.

To date, the lateral diffusion at the neuronal surface has been demonstrated for glutamatergic metabotropic mGlu5, AMPA, and NMDA receptors (56-60), glycine receptor $(61,62)$, GABA-A receptor (63), $\beta 2$-adrenergic receptor (64), $\mu$-opioid receptor (65), and, finally, D1R (66). This initial demonstration of D1R mobility showed that the majority of dendritic D1Rs diffuse in the plane of the membrane and that their mobility is confined by interaction with NMDAR (66). Exposure to NMDA reduced the diffusion coefficient for D1R and caused an increase in the number of D1Rpositive spines (66), a situation highly reminiscent of the elevated extracellular levels of glutamate $(12)$ and DA $(13,41)$ associated with LID. Such interaction could be direct (66) or indirect through the PSD-95 protein that interacts and regulates D1R trafficking and anchorage $(26,27,42)$. Psychostimulants in wild-type mice or genetic models of hyperdopaminergia have shown reduced PSD-95 levels in the striatum $(42,50)$. In this hyperdopaminergic context, PSD-95 inhibits the physical association between D1 and NMDAR and functionally uncouples D1R trafficking and signaling from modulation by NMDAR (28). In the L-DOPA-exposed dopaminedepleted experimental condition, the situation was rather different, with anchorage of D1R under the control of PSD-95.

To our knowledge, however, the role of surface receptor lateral diffusion in such a pathological condition had not previously been determined. We therefore addressed the dynamics of surface synaptic D1R by single QD tracking (48) to discern the mechanisms underlying PSD-95-dependent D1R trafficking. In keeping with the initial report (66), surface D1Rs were mobile and diffusive in PSD-95-expressing neurons. However, lowering PSD-95 levels using shPSD further increased the overall surface diffusion of D1R and decreased D1R surface membrane localization, but did not affect GluN2B-NMDAR surface expression. Comparable effects were obtained with the TAT-D1/PSD-95 peptide, which prevents direct interaction between D1R and PSD-95. However, it is important to note that knocking down PSD-95 had a broader effect on D1R surface dynamics than did TAT-D1-CT. It is thus likely that other interacting partners of PSD-95 participate in the membrane organization of D1R.

This latter point further supports the claim of a direct role of PSD-95 in governing the surface stabilization of D1R in the extrasynaptic compartment, as well as in the vicinity of corticostriatal synapses. Both diminishing PSD-95 expression levels and competing with direct interaction between D1R and PSD-95 clearly favored D1R lateral diffusion, possibly by unanchoring D1R in dendritic spines. In addition, PSD-95 can alter D1R intracellular trafficking (exocytotic or endocytotic rate) and surface content $(26,27,42)$, which consequently modulates the dynamics of the surface receptors. For instance, D1Rs form dimers with GluN1/ GluN2A containing NMDA receptors (67), an association that is regulated by PSD-95 (28) and reduced after chronic L-DOPA treatment (68). Diminishing PSD-95 expression levels or competing with direct interaction between D1R and PSD-95 unanchors D1R, but would thus favor reformation of D1/NMDA complexes, a dynamic that warrants investigation.

We posited that releasing pathologically anchored D1R from dendritic spines would contribute to reduced LID. Our rat and primate experiments support this claim, with dramatic reductions in LID severity and concomitant increases in Good On-time (i.e., time without disabling dyskinesia). Development of such strategy should not, however, interfere with the antiparkinsonian action of the gold standard therapy, L-DOPA. In that respect, our nonhuman primate experiments clearly showed that lowering PSD-95 levels had no negative effect on therapeutic response to L-DOPA. Glutamatergic corticostriatal transmission seemed to be preserved, as further supported by our pharmacological experiments with amantadine. At present, the noncompetitive NMDA receptor antagonist amantadine is frequently used as adjunctive therapy for $\operatorname{LID}(43,69,70)$. Combination of L-DOPA and amantadine in shPSD animals caused near-complete suppression of dyskinesia and Bad On-time, dramatically increasing Good Ontime. Together, these data suggest that NMDA-mediated corticostriatal transmission is not blunted in the shPSD animals, as they additively benefited from combination therapy with L-DOPA and amantadine. Rather, combined knockdown of striatal PSD-95 expression and amantadine therapy may be superior to amantadine alone as an adjunct to L-DOPA to treat dyskinetic side effects in PD patients.

Our data set identified a pathological mechanism for D1R anchoring at synapses. Pathological overexpression of PSD95 anchors D1R in the dopamine-depleted brain, reducing its mobility in and out of synapses. Easing such mobility through enhanced lateral diffusion is achieved by (a) lowering PSD95 expression levels and (b) administration of cell-permeable peptide affecting D1/PSD-95 interaction, with a magnitude compatible with preservation of corticostriatal communication Because alterations in D1R function contribute to the clinical features of PD and underlie the development of dyskinesia, therapies aiming at interrupting or regulating its trafficking via synapse-associated scaffolding protein organization, such as the cell-permeable peptide tested in the present study, may be useful in the treatment of dyskinesia in PD patients. Thus, understanding the rules that govern D1R surface distribution and, most importantly, their dynamic retention in postsynaptic density may shed new light on the fine-tuning of D1R in pathological conditions such as PD and LID.

\section{Methods}

\section{Rat experiments}

$L V$ construction. We used $4 \mathrm{LVs}$, provided and validated by O.M. Schlüter (Stanford University School of Medicine, Palo Alto, California, USA; ref. 36). The different sequences were cloned into the LV vector FUGW (35), used as control (mock). The 3 other vectors were FUGW plus PSD-95, FUG plus shRNA for PSD-95, and FUGW plus shRNA for PSD-95 plus PSD-95 (referred to herein as PSD, shPSD, and SH+PSD, respectively). LV production was performed in the IFR 66 Vectorology Platform (University of Bordeaux 2, Bordeaux, France) by transfection with a 3 viral vector 
system - mock (with the different inserts), pCMV- $\Delta 8-9$ (encapsidation plasmid), and VSV-G (cDNA encoding the envelope glycoprotein of vesicular stomatitis virus) - in FT-HEK293 cells. LV supernatants were concentrated by centrifugation concentration filter (centricon) to obtain a final titer of $2.5 \times 10^{9} \mathrm{pI} / \mathrm{ml}$.

Peptide preparation and validation. We used active peptides (D1-CT) corresponding to the last 46-amino acid sequence (from Leu401 to Thr446) of rat D1R and a scrambled version of the peptide as negative control (D1-SCR) (GenScript). Biotinylated forms of peptides were used for pulldown assays. For functional experiments, we used FITC-tagged D1 peptides fused to the cell membrane transduction domain of the human immunodeficiency virus 1 (YGRKKRRQRRR) to gain cell permeability (71). Peptides were dissolved immediately before used in water $(10 \mathrm{mg} / \mathrm{ml})$ for pulldown assays and in PBS at their final concentration for in vivo intracerebral administration.

For pulldown assay, $500 \mathrm{~g}$ of protein extracts from rat or monkey brain tissues as well as HEK293 cells transfected with PSD-95 or PSD-95-GFP were incubated with immobilized streptavidin bound to the indicated biotinylated peptides $(100 \mu \mathrm{g})$ overnight at $4^{\circ} \mathrm{C}$, as previously described $(72)$. Beads $(40 \mu \mathrm{l})$ were washed 4 times with $150 \mathrm{mM} \mathrm{NaCl}$ buffer and eluted with 1 volume of SDS sample buffer. Eluates $(20 \mu \mathrm{l})$ and input samples $(10 \mu \mathrm{g})$ were subjected to SDS-PAGE and Western blot using mouse antiPSD-95 (UC Davis/NIH NeuroMab Facility) as described below.

Stereotactic procedures. Adult male Sprague Dawley rats (Charles River Laboratories), weighing 175-200 g at the beginning of the experiment, were used. They were maintained under standard laboratory conditions in a 12-hour light/12-hour dark cycle with free access to food and water. The 6-OHDA lesion was performed as previously described (14). Unilateral DA deprivation of the striatum was obtained by injection with 6-OHDA ( $3 \mu \mathrm{g} / \mu \mathrm{l}$; Sigma-Aldrich) in the right medial forebrain bundle $(2.5 \mu \mathrm{l}$ at anterior-posterior $-3.7 \mathrm{~mm}$, medial-lateral $1.7 \mathrm{~mm}$, and dorsoventral $-8 \mathrm{~mm}$ relative to bregma; refs. 41,73$)$ in rats pretreated with citalopram (1 mg/kg i.p.; Lundbeck), an inhibitor of serotonin reuptake, and with desipramine hydrochloride ( $20 \mathrm{mg} / \mathrm{kg}$ i.p.; Sigma-Aldrich), an inhibitor of noradrenergic reuptake (74). $5 \mu \mathrm{l}$ of the concentrated LVs (mock, PSD$95, \mathrm{SH}+\mathrm{PSD}$, and shPSD) or $3 \mu \mathrm{l}$ of peptide solution was injected into the caudal lesioned putamen at the following coordinates: anterior-posterior, 0.2; medial-lateral, 3.5; dorsoventral, 5.7. LVs were injected either at the time of the 6-OHDA lesion (set 1) or after behavioral pretesting (set 2) (Supplemental Figure 1), while the peptides were injected concomitantly with L-DOPA on testing days.

Behavioral assessment. Animals displaying both an impaired stepping test measured on days 18-20 (75-77) and >95\% loss of TH-immunopositive fibers in the striatum $(78,79)$, assessed after completion of all experiments, were retained for final analysis (Supplemental Figure 1). 3 weeks after 6-OHDA injection, rats were treated once daily with benserazide (15 mg/kg i.p.; Sigma-Aldrich) or L-DOPA ( $6 \mathrm{mg} / \mathrm{kg}$ i.p.; Sigma-Aldrich) and benserazide ( $15 \mathrm{mg} / \mathrm{kg}$ i.p.) for 9 days in order to induce gradual AIM development ( $n=9$ [mock]; 12 [PSD-95]; 8 [SH+PSD and shPSD]). In the second set of experiments, the rats were first treated with L-DOPA for 10 days with daily testing for AIMs. LV was injected on day 12 , and rats were allowed to recover for 9 days before resuming the injection schedule and then tested for AIMs for the next 7 days $(n=9$ [mock and shPSD]; 13 [PSD-95]; 11 [SH+PSD]). Peptide experiments were conducted on 11 dyskinetic rats. The 4 AIM categories (limb, axial, orolingual, and locomotive) were scored using a validated rating scale $(33,34)$ for 1 minute every 20 minutes for 2 hours (total 6 observations; maximal score for each observation, 16; maximal total score per session, 96) by a trained investigator as described previously $(41,80)$. Figures display the sum of the axial, limb, and orolingual AIMs.
Upon completion of the behavioral testing schedule, rats were deeply anesthetized with sodium chloral hydrate $(400 \mathrm{mg} / \mathrm{kg}) 1$ hour after L-DOPA injection and killed either by decapitation (for Western blotting experiments), with their brains being rapidly removed and frozen in a Snap Frost 80 Specimen Freezing System (Alphelys), or by transcardiac perfusion (for immunohistochemistry experiments) with 50-100 $\mathrm{ml} 0.9 \%$ $\mathrm{NaCl}$ followed by $250 \mathrm{ml}$ 4\% PFA in $0.1 \mathrm{M}$ phosphate buffer ( $\mathrm{pH}$ 7.4), with their brains being removed and postfixed overnight in $4 \%$ PFA at $4{ }^{\circ} \mathrm{C}$.

Western blot. Patches of tissue were collected directly around the injection site from cryostat-cut $200-\mu \mathrm{m}$-thick sections after GFP expression signal was observed immediately before and after on adjacent $20-\mu \mathrm{m}$-thick sections under fluorescence microscopy to delineate the transfected area. Mirror contralateral patches were collected as controls. Patches were individually sonicated in a lysis buffer RIPA (R0278; Sigma Aldrich) containing $0.1 \%$ SDS and centrifuged at $20,817 \mathrm{~g}$ at $4^{\circ} \mathrm{C}$ for 15 minutes. The resulting supernatants were collected, and the proteins were reduced with a buffer containing $25 \mathrm{mM}$ DTT and $1 \%$ SDS until quantification of the whole protein by the Lowry method (Bio-Rad DC Protein Assay). Samples containing $10 \mu \mathrm{g}$ of proteins were heated to $100^{\circ} \mathrm{C}$ for 5 minutes, and electrophoresis was performed onto a $8 \%$ polyacrylamide gel. Proteins were blotted on polyvinylidene fluoride membranes (Immobilon P; Millipore) in Miniprotean III (BioRad). Membranes were saturated for 90 minutes by incubation with 5\% milk in TBS-Tween (Tris HCL, pH 7.5; 100 mM NaCl; 0.1\% Tween 20 ) and incubated overnight with the following primary monoclonal antibodies diluted in TBS-Tween $0.1 \%$ supplemented with $1 \%$ BSA: anti-PSD95 (UC Davis/NIH NeuroMab Facility), anti-tubulin (Sigma-Aldrich), anti-GFP (Invitrogen), anti-phosphoERK1/2 (Sigma-Aldrich) and antiERK1/2 (Cell Signalling Technology). Membranes were then incubated for 2 hours at room temperature with secondary anti-mouse or anti-rabbit (both 1:5,000; Jackson ImmunoResearch) HRP-coupled antibody diluted in TBS-Tween $0.1 \%$ and supplemented with $5 \%$ milk. The complexes were detected with enhanced chemiluminescence reagents (Eurobio). Images were acquired on a computer with a CCD camera coupled with an optical bench. Optical density (OD) was measured with Mercator software (Explora Nova). Results were derived from 3 independent experiments.

Immunohistochemistry. Cryostat-cut $50-\mu \mathrm{m}$-thick free-floating sections were processed for PSD-95/GFP double immunohistochemistry. Block step in 5\% milk powder in PBS for 30 minutes was followed by overnight incubation at room temperature with a mixture of mouse monoclonal antibody to PSD-95 (73 028; UC Davis/NIH NeuroMab Facility) and rabbit polyclonal antibody to GFP (A11122; Invitrogen) diluted at 1:2,000 and $1: 1,000$, respectively, in $0.2 \%$ BSA, $0.3 \%$ triton X100, and PBS. PSD-95 revelation was conducted first, and sections were incubated with anti-mouse HRP envision system (K4001; Dako) for 30 minutes at room temperature, rinsed in PBS, and incubated in anti-HRP 549 dye light conjugate diluted 1:1,000 in PBS for 60 minutes at room temperature. Sections were then incubated in anti-rabbit Alexa Fluor 488 diluted 1:500 in PBS for $60 \mathrm{~min}$ at room temperature for revealing GFP signal. Sections were mounted on gelatin-coated slides in PBS and coverslipped with Vectashield HardSet mounting medium (H1400; Vector Laboratories). Fluorescent images were captured on a confocal Leica microscope DM 2500 TCS SPE. Digital images were acquired separately for each wavelength. Adobe Photoshop CS4 was used to further process digital images. Any adjustments to brightness and contrast were made uniformly to all parts of the image.

Cytochrome oxidase subunit I (COX I) qPCR. RNA extracts from isolated SNr were purified with RNeasy Plus Micro Kit (Qiagen), and $10 \mathrm{ng}$ was retrotranscribed to cDNA at $42^{\circ} \mathrm{C}$ with reverse transcriptase (Fermentas) in the presence of $1 \mu \mathrm{l}$ random primer $(150 \mathrm{ng} / \mu \mathrm{l})$ and $1 \mu \mathrm{l}$ oligodt $(200 \mathrm{ng} / \mu \mathrm{l})$. Real-time PCR was performed on a Light Cycler 480 (Roche) in a total volume of $10 \mu \mathrm{l}$ using $1 \mathrm{ng}$ cDNA as tem- 
plates, $3 \mu \mathrm{l}$ mixed forward (GCTGGCTTCGTCCACTGATT) and reverse (AAGTGGGCTTTTGCTCATGTG) primers, and $5 \mu$ of mix 2 X Roche containing Fast Start Taq DNA polymerase reaction buffer dNTP mix (with dUTP instead of dTTP), Sybr Green I dye, and $\mathrm{MgCl}_{2}$. Reliability of reference genes (eEf1a1 and Sdha) was evaluated with GeNorm. The cycle point $(\mathrm{CP})$ value of COX I was normalized against that of Sdha and Eef1a1, and the relative level of expression was calculated using the comparative $2^{-\Delta \Delta \mathrm{Ct}}$ method (81).

\section{Monkey experiments}

Animals. 37 F2-bred female rhesus monkeys (Macaca mulatta, Xierxin; mean weight, $5.3 \pm 0.8 \mathrm{~kg}$; mean age, $5 \pm 1$ years) were used. Animals were housed in individual primate cages under controlled conditions of humidity $(50 \%$ $\pm 5 \%$ ), temperature $\left(24^{\circ} \mathrm{C} \pm 1{ }^{\circ} \mathrm{C}\right)$, and light (12-hour light/12-hour dark cycles, lights on at 8:00 AM), with food and water ad libitum. Veterinarians skilled in the healthcare and maintenance of nonhuman primates supervised animal care. All reasonable efforts were made to minimize animal suffering. The use of primates was minimized by using an experimental design that permits statistically significant changes to be demonstrated with the smallest number of animals per group and the smallest number of groups, consistent with scientific rigor. Experiments followed previously published procedures $(18,22,25,37,82)$.

Experimental protocol. See Supplemental Figure 1. 6 animals were kept as untreated controls (control group), and 6 received $20 \mathrm{mg} / \mathrm{kg}$ L-DOPA twice daily for 3 months (control chronic L-DOPA). The remaining 25 animals were treated daily at 9:00 AM with MPTP hydrochloride $(0.2 \mathrm{mg} / \mathrm{kg}$ i.v.; Sigma-Aldrich) dissolved in saline according to a previously described protocol (83). Following stabilization of the MPTPinduced syndrome, animals received saline (MPTP group; $n=6$ ) or $20 \mathrm{mg} / \mathrm{kg}$ L-DOPA twice daily for 3 months (MPTP chronic L-DOPA group; $n=19$ ). 13 animals developed severe and reproducible dyskinesia, presenting choreic-athetoid (characterized by constant writhing and jerking motions), dystonic, and sometimes ballistic movements (large-amplitude flinging, flailing movements) (MPTP-intoxicated and chronically L-DOPA-treated, dyskinetic group), whereas 6 others did not (MPTP-intoxicated and chronically L-DOPA-treated, nondyskinetic group). The MPTP intoxication protocol, chronic L-DOPA treatment, clinical assessments, terminal procedure, and characterization of the extent of nigrostriatal denervation in the animals terminated before viral transfection were conducted as previously published $(18,22,25,37,82)$. DAT binding autoradiography using [125 I]-(E)-N-(3-iodoprop-2-enyl)$2 \beta$-carboxymethyl-3 $\beta$-(4'-methylphenyl)-nortropane (Chelatec) showed a dramatic and similar reduction (>95\%) in all MPTP-treated groups compared with control animals, as published elsewhere (84).

7 dyskinetic chronically L-DOPA-treated MPTP-intoxicated animals were kept alive for further behavioral investigation of PSD-95 knockdown. Monkeys' response to their tailored dose of L-DOPA/carbidopa (ranging $15-20 \mathrm{mg} / \mathrm{kg}$ for maximal reversal of PD symptoms) was defined prior to LV intracerebral injections. The improved Horsley-Clarke stereotactic technique was used as previously described to inject, at 2 rostrocaudal levels of the motor striatum (anterior commissural, $-1 \mathrm{~mm}$ and $-5 \mathrm{~mm}$ ), $100 \mu \mathrm{l}$ $\operatorname{shPSD}(n=4)$ or GFP (mock; $n=3) \operatorname{LV}(25,37,85)$. Starting at 4 weeks after surgery, animals were repeatedly assayed 2 days apart for behavioral responses to $100 \%$ of the tailored oral dose of L-DOPA, alone or in combination with amantadine (20 mg/kg orally) (44). The monkeys' behavior was first recorded in the Off state for 60 minutes in an observation cage $(1.1 \mathrm{~m} \times 1.5 \mathrm{~m} \times 1.1 \mathrm{~m})$. Drugs were then administered, and behavior was recorded for a further 240 minutes in the observation cage. The total duration of observation was 300 minutes, including drug administration. The parkinsonian condition (and its reversal) was assessed on a parkinsonian monkey rating scale using videotaped recordings of monkeys. A score of 0 corresponded to a normal animal; a score above 6 corresponded to a parkinsonian animal. The severity of dyskinesia was rated using the dyskinesia disability scale: 0 , dyskinesia absent; 1 , mild, fleeting, and rare dyskinetic postures and movements; 2 , moderate, more prominent abnormal movements, not interfering substantially with normal behavior; 3 , marked, frequent, and at times continuous dyskinesia, intruding on the normal repertoire of activity; 4 , severe, virtually continuous dyskinetic activity replacing normal behavior and disabling to the animal. Locomotor activity was concomitantly monitored with infrared activity monitors, providing a mobility count every 5 minutes. The duration of antiparkinsonian action (i.e., On-time) was defined as the number of minutes for which bradykinesia was absent (score of 0 ). In addition, the duration of On-time associated with dyskinesia of varying severity was defined as follows: Good On-time, number of minutes for which bradykinesia was 0 and dyskinesia was either absent or of mild or moderate severity (score of 0-2); Bad On-time, number of minutes for which bradykinesia was 0 and dyskinesia was either marked or severe (score of 3-4).

Recombinant shRNA LV plasmids and virus production. shRNA LV plasmids were purchased from Sigma-Aldrich. shRNAs were validated based on the Genbank human PSD-95 sequence NM001365. All sequences were BLAST confirmed for specificity. Synthetic DNA encoding shRNA sequences targeting human PSD-95 were cloned into shRNA LV plasmids (pLKO.1-puro) under the control of the U6 promoter. shRNA LV plasmids were transfected into SH-SY5Y cells for 48 hours to determine the level of knockdown of gene expression by immunoblotting. The shRNA sequence used for this study was CCGGACGATCATCGCTCAGTATAAACTCGAGTTTATACTGAGCGATGATCGTTTTTTG. The GFP, scramble shRNA, and PSD-95 shRNA LVs were tested as described above. $\mathrm{LV}$ titers were $8.67 \times 10^{8} \mathrm{pI} / \mathrm{ml}$ for PSD-95 shRNA, $1.03 \times 10^{9}$ for scramble shRNA, and $2.00 \times 10^{9}$ for GFP.

Coimmunoprecipitation of endogenous D1 with anti-PSD-95 antibody. Punches of striatum from the dyskinetic chronically L-DOPA-treated MPTPintoxicated monkeys $(n=6)$ were collected and homogenized in RIPA buffer (Sigma-Aldrich) with a cocktail of protease inhibitors (Roche). Homogenates $(300 \mu \mathrm{g})$ were incubated with mouse anti-PSD-95 (UC Davis/NIH NeuroMab Facility) or rabbit anti-D1 (86) antibodies at $4{ }^{\circ} \mathrm{C}$ overnight with $30 \mu$ l of protein G-sepharose (GE Healthcare). Beads were washed 4 times with TBS $(50 \mathrm{mM}$ Tris and $150 \mathrm{mM} \mathrm{NaCl})$ and eluted in SDS sample buffer. Coprecipitated proteins were then identified by SDSPAGE and Western blot analysis using mouse anti-PSD-95 (UC Davis/NIH NeuroMab Facility) or rat anti-D1 (Sigma-Aldrich) as indicated. Signals were revealed with HRP-conjugated secondary antibodies and chemiluminescence. Results were derived from 3 independent experiments.

Immunohistochemistry. PSD-95 Western blotting was identical to the method described above for fresh-frozen rat tissue ( $n=6$ per experimental condition). Results were derived from 3 independent experiments. For transfected animals, free-floating sections were chosen directly in the injection site. Putamen was dissected from these sections, pretreated with proteinase $\mathrm{K}$ (Dako), and sonicated in $100 \mu \mathrm{l}$ Laemmli buffer $(25 \mathrm{mM}$ Tris-Cl, pH 6.8; 1\% SDS; 250 mM DTT; 7.5\% glycerol; 0.05\% bromophenol blue), $20 \mu \mathrm{l}$ of which was analyzed by PSD-95 and tubulin immunoblot as described above. Striatum (PSD-95) and SNc (TH; 1 every 12 sections) cryostat free-floating sections $(50 \mu \mathrm{m})$ were incubated overnight in mouse monoclonal antibody to PSD-95 (1:2,000 in BSA; UC Davis/NIH NeuroMab Facility) and TH (1:10,000 in BSA; Millipore), respectively. Signals were revealed with the HRP anti-mouse Envision system (Dako) followed by DAB chromogen reaction (Vector Laboratories). OD of PSD-95 staining was measured in delimited striatum areas (Mercator, Explora Nova). Stereological unbiased counting of TH-positive neurons in delimited SNc 
was estimated with Mercator software using a Leica CTR6000 microscope ( $\times 40$ objective). The optical fractionator option was used, with a probe size of $100 \mu \mathrm{m} \times 80 \mu \mathrm{m}$ spaced by $600 \mu \mathrm{m} \times 400 \mu \mathrm{m}$.

\section{Rat striatal primary culture experiments}

Neuronal preparation and cell transfection. Rat striatal cultures were prepared from embryonic day 15 rat brains as previously described (87-89). All the reagents for the culture were purchased from Sigma-Aldrich and Invitrogen. Cells were plated on coverslips coated with poly-D-lysine $(10 \mu \mathrm{g} / \mathrm{ml})$ and laminin $(10 \mu \mathrm{g} / \mathrm{ml})$ at a density of 150,000 per dish. Striatal cultures were grown in Neurobasal medium (Invitrogen) and supplemented with B27 (Invitrogen), $0.5 \mathrm{mM}$ glutamine, and $12.5 \mu \mathrm{M}$ glutamate. Before seeding, coverslips were incubated with fetal calf serum-supplemented $20 \%$ Dulbecco modified Eagle medium/F12 for 1 hour at $37^{\circ} \mathrm{C}$. After seeding, the cells were kept at $37^{\circ} \mathrm{C}$ in a $5 \% \mathrm{CO}_{2}$ incubator for 10 days. At 4 days in vitro, part of the medium, together with nonadherent cells and cell debris, was removed, and culture medium with cytosine arabinofuranoside $(0.5 \mu \mathrm{M})$ and without glutamate was added. Striatal neurons were transfected at 7 days in vitro with D1R-CFP and PSD-95-GFP or with shPSD-GFP alone using the Effectene transfection kit (Qiagen). For 4 wells, we mixed $1.2 \mu \mathrm{g}$ of DNA with $24 \mu \mathrm{l}$ effectene and $9.6 \mu \mathrm{l}$ enhancer in $300 \mu \mathrm{l}$ reaction buffer, then added the mixture to cultured neurons, which were transferred to new culture medium 10 minutes beforehand. After a 45-minute incubation, neurons were placed back in their original medium.

Single $Q D$ tracking and surface diffusion. As previously described (48, 59, 60, 90, 91), QDs 655 Goat F(ab')2 anti-Rabbit IgG (Invitrogen) were first incubated for 30 minutes with $1 \mu \mathrm{l}$ polyclonal antibodies against GFP (Chemicon). Nonspecific binding was blocked by additional casein (Vector Laboratories) to the QD 15 minutes before use. Cultured neurons were then incubated for 10 minutes with precoated anti-GFP QD complexes (final dilution, 1:10,000) and mounted in a heated chamber for observation. D1R-QDs were detected using a mercury lamp and appropriate excitation/emission filters. Images were obtained with an acquisition time of $50 \mathrm{~ms}$ with up to 1,000 consecutive frames at an acquisition rate of 30 $\mathrm{Hz}$. Signals were detected using an EMCCD camera (Quantem, Roper Scientific). QDs were followed on randomly selected dendritic regions for up to 30 minutes. QD recording sessions were processed using Metamorph software (Universal Imaging Corp.). The instantaneous diffusion coefficient $D$ was calculated for each trajectory, from linear fits of the first 4 points of the mean square displacement versus time $[\mathrm{MSD}(t)]$ function, using $\operatorname{MSD}(t)=\left\langle r^{2}\right\rangle(t)=4 D t$. The 2-dimensional trajectories of single molecules in the plane of focus were constructed by correlation analysis between consecutive images using a Vogel algorithm.

Immunocytochemistry and image analysis. For immunostaining, surface D1R-CFP or GluN2B were specifically stained using a rabbit polyclonal antibody raised against GFP (1:200; Invitrogen) or a rabbit polyclonal antibody raised against GluN2B with extracellular epitope (1:100; Alomone) for 15 minutes on live neurons at $37^{\circ} \mathrm{C}$ in culture medium. Briefly, neurons were then fixed with 4\% PFA for 15 minutes, washed, and incubated with secondary antibody anti-rabbit Alexa Fluor 568 (1:1,000 for 30 minutes; Molecular Probes). For PSD-95 and total D1R immunostaining, we used a monoclonal antibody raised in mouse antiPSD-95 (1:500 for 30 minutes at room temperature; UC Davis/NIH NeuroMab Facility) or a monoclonal antibody raised in mouse anti-D1R (1:500 for 60 minutes at $37^{\circ} \mathrm{C}$; Invitrogen). Cultures were treated with a $37^{\circ} \mathrm{C}$ prewarmed mixture of $4 \%$ paraformaldehyde in $0.1 \mathrm{M} \mathrm{PB}(\mathrm{pH}$ 7.4) for 20 minutes at $14^{\circ} \mathrm{C}$, rinsed 3 times in PBS, and permeabilized with $0.3 \%$ Triton X-100 in PBS for 50 seconds at room temperature. The cultures were then incubated in PBS with $0.3 \%$ BSA (Sigma-Aldrich) for 60 minutes at room temperature to block nonspecific binding sites.
Coverslips were placed cell-side-down onto a 200- $\mu 1$ drop of primary antibodies (in PBS-BSA). Cultures were washed 3 times in PBS-BSA and incubated at room temperature for 30-60 minutes with a secondary Alexa Fluor 568-conjugated goat anti-mouse IgG (1:400 in PBS for D1R, 1:1,000 for PSD-95; Invitrogen). For D1R immunostaining, coverslips were further incubated with Alexa Fluor 568-conjugated donkey antigoat immunoglobulin G (1:400 in PBS; Invitrogen) to increase the fluorescence signal (88). The slides were then rinsed and mounted in Vectashield (Vector Laboratories), and preparations were kept at $4{ }^{\circ} \mathrm{C}$ until quantification. For analysis, immunofluorescent stainings were imaged on an inverted confocal spinning-disk microscope (Leica). Fluorescence intensity was measured using Metamorph software (Universal Imaging) and corrected for background noise in the different conditions. Surface D1R was measured on $n=5$ neurons (i.e., $N=21$ dendritic fields) in the PSD-95 condition, and on $n=6$ neurons (i.e., $N=20$ dendritic fields) in the shPSD condition.

\section{Statistics}

Data are expressed as mean \pm SEM unless otherwise specified. Analysis involving comparisons of 2 groups were performed using 2-tailed unpaired $t$ test. Western blotting experiments involving several experimental groups were analyzed using 1-way ANOVA followed by Dunnett. Behavioral data (rating scores and locomotor activity) in rat and macaque models were analyzed using Kruskal-Wallis followed by Dunn's multiple-comparisons test. QD experiments, reporting diffusion coefficient distribution, were analyzed using Mann-Whitney test. Analyses were completed using STATA (Intercooled Stata 6.0; Stata Corp.). A $P$ value less than 0.05 was considered significant.

\section{Study approval}

All experiments were carried out in accordance with the European Communities Council Directive of November 24, 1986 (86/609/EEC). The Ethical Committee of CNRS, Région Aquitaine (Bordeaux, France), approved the rat experiments. Monkey experiments were performed in an AAALACaccredited facility following acceptance of study design by the Institute of Lab Animal Science IACUC (Chinese Academy of Science, Beijing, China).

\section{Acknowledgments}

This work was supported by Agence Nationale de la Recherche grants ANR-07-MNP TRAFINLID and ANR-08-MNP-018 MCHPRIMAPARK (to B. Bloch and E. Bezard), by a Federation pour la Recherche sur le Cerveau grant (to E. Bezard), by Biothèque Primate - CNRS Life Sciences Department (to E. Bezard), by Conseil Régional d'Aquitaine (to A. Berthet, E. Bezard, L. Groc, and D. Choquet), by a postdoc fellowship from Fondation pour la Recherche Médicale (to B. Dehay), by ERC grant 232942 (to D. Choquet), and by FP7-PEOPLE-2009-RG (to B. Dehay). We thank the Bordeaux Imaging Center for technical assistance, B. Rousseau and L. Hao for animal care, M.-L. Thiolat for general technical assistance, and V. Guyonnet-Dupeyrat (Université Bordeaux Segalen Vectorology Core facility) and T. Leste-Lasserre, G. Drutel, and A. Brochard (Université Bordeaux Segalen qPCR Core facility). Université Bordeaux Segalen and CNRS provided infrastructural support.

Received for publication May 2, 2012, and accepted in revised form August 16, 2012.

Address correspondence to: Erwan Bezard, CNRS UMR 5293, IMN, Université Bordeaux Segalen, 146 rue Léo Saignat, 33076 Bordeaux cedex, France. Phone: 5575.71687; Fax: 5569.86182; E-mail: erwan.bezard@u-bordeaux2.fr. 
1. Carr DB, Sesack SR. Hippocampal afferents to the rat prefrontal cortex: synaptic targets and relation to dopamine terminals. J Comp Neurol. 1996; 369(1):1-15.

2. Gerfen CR. The neostriatal mosaic: multiple levels of compartmental organization. J Neural Transm Suppl. 1992;36:43-59.

3. Calabresi P, Picconi B, Tozzi A, Di Filippo M. Dopamine-mediated regulation of corticostriatal synaptic plasticity. Trends Neurosci. 2007;30(5):211-219.

4. Centonze D, et al. Subthalamic nucleus lesion reverses motor abnormalities and striatal glutamatergic overactivity in experimental parkinsonism. Neuroscience. 2005;133(3):831-840.

5. Chase TN, Oh JD, Konitsiotis S. Antiparkinsonian and antidyskinetic activity of drugs targeting central glutamatergic mechanisms. J Neurol. 2000;247(suppl 2):II36-II42.

6. Fahn S. How do you treat motor complications in Parkinson's disease: Medicine, surgery, or both? Ann Neurol. 2008;64(suppl 2):S56-S64

7. Obeso JA, et al. The origin of motor fluctuations in Parkinson's disease: importance of dopaminergic innervation and basal ganglia circuits. Neurology. 2004;62(1 suppl 1):S17-S30.

8. Rascol O. Medical treatment of levodopa-induced dyskinesias. Ann Neurol. 2000;47(4 suppl 1):S179-S188.

9. Picconi B, et al. Abnormal Ca2+-calmodulindependent protein kinase II function mediates synaptic and motor deficits in experimental parkinsonism. J Neurosci. 2004;24(23):5283-5291.

10. Gardoni F, Di Luca M. New targets for pharmacological intervention in the glutamatergic synapse. Eur J Pharmacol. 2006;545(1):2-10.

11. Hallett PJ, Spoelgen R, Hyman BT, Standaert DG, Dunah AW. Dopamine D1 activation potentiates striatal NMDA receptors by tyrosine phosphorylation-dependent subunit trafficking. J Neurosci. 2006;26(17):4690-4700.

12. Robelet S, Melon C, Guillet B, Salin P, KerkerianLe Goff L. Chronic L-DOPA treatment increases extracellular glutamate levels and GLT1 expression in the basal ganglia in a rat model of Parkinson's disease. Eur J Neurosci. 2004;20(5):1255-1266.

13. Lindgren HS, Andersson DR, Lagerkvist S, Nissbrandt $\mathrm{H}$, Cenci MA. L-DOPA-induced dopamine efflux in the striatum and the substantia nigra in a rat model of Parkinson's disease: temporal and quantitative relationship to the expression of dyskinesia. J Neurochem. 2010;112(6):1465-1476

14. Berthet A, et al. Pharmacological analysis demonstrates dramatic alteration of D1 dopamine receptor neuronal distribution in the rat ana$\log$ of L-DOPA-induced dyskinesia. J Neurosci. 2009;29(15):4829-4835.

15. Guigoni C, Doudnikoff E, Li Q, Bloch B, Bezard E. Altered $D(1)$ dopamine receptor trafficking in parkinsonian and dyskinetic non-human primates. Neurobiol Dis. 2007;26(2):452-463.

16. Hallett PJ, et al. Alterations of striatal NMDA receptor subunits associated with the development of dyskinesia in the MPTP-lesioned primate model of Parkinson's disease. Neuropharmacology. 2005;48(4):503-516.

17. Silverdale MA, et al. Synaptic recruitment of AMPA glutamate receptor subunits in levodopa-induced dyskinesia in the MPTP-lesioned nonhuman primate. Synapse. 2010;64(2):177-180.

18. Aubert I, et al. Increased D1 dopamine receptor signaling in levodopa-induced dyskinesia. Ann Neurol. 2005;57(1):17-26.

19. Picconi B, et al. Loss of bidirectional striatal synaptic plasticity in L-DOPA-induced dyskinesia. Nat Neurosci. 2003;6(5):501-506.

20. Santini E, et al. Critical involvement of cAMP/ DARPP-32 and extracellular signal-regulated protein kinase signaling in L-DOPA-induced dyskinesia. JNeurosci. 2007;27(26):6995-7005.
21. Westin JE, Vercammen L, Strome EM, Konradi C, Cenci MA. Spatiotemporal pattern of striatal ERK1/2 phosphorylation in a rat model of L-DOPAinduced dyskinesia and the role of dopamine D1 receptors. Biol Psychiatry. 2007;62(7):800-810.

22. Santini E, et al. Distinct changes in cAMP and extracellular signal-regulated protein kinase signalling in L-DOPA-induced dyskinesia. PloS One. 2010;5(8):e12322.

23. Cenci MA, Lindgren HS. Advances in understanding L-DOPA-induced dyskinesia. Curr Opin Neurobiol. 2007;17(6):665-671.

24. Cenci MA, Lundblad M. Post- versus presynaptic plasticity in L-DOPA-induced dyskinesia. J Neurochem. 2006;99(2):381-392.

25. Ahmed MR, et al. Lentiviral overexpression of GRK6 alleviates L-dopa-induced dyskinesia in experimental Parkinson's disease. Sci Transl Med. 2010; 2(28):28ra28.

26. Fiorentini C, Gardoni F, Spano P, Di Luca M, Missale C. Regulation of dopamine D1 receptor trafficking and desensitization by oligomerization with glutamate $\mathrm{N}$-methyl-D-aspartate receptors. J Biol Chem. 2003;278(22):20196-20202.

27. Yao WD, Spealman RD, Zhang J. Dopaminergic signaling in dendritic spines. Biochem Pharmacol. 2008;75(11):2055-2069.

28. Zhang J, et al. PSD-95 uncouples dopamine-glutamate interaction in the D1/PSD-95/NMDA receptor complex. J Neurosci. 2009;29(9):2948-2960.

29. Nash JE, Johnston TH, Collingridge GL, Garner CC, Brotchie JM. Subcellular redistribution of the synapse-associated proteins PSD-95 and SAP97 in animal models of Parkinson's disease and L-DOPAinduced dyskinesia. FASEB J. 2005;19(6):583-585.

30. Bezard E, Brotchie JM, Gross CE. Pathophysiology of levodopa-induced dyskinesia: potential for new therapies. Nat Rev Neurosci. 2001;2(8):577-588.

31. Jenner P. Molecular mechanisms of L-DOPAinduced dyskinesia. Nat Rev Neurosci. 2008; 9(9):665-677.

32. Berthet A, Bezard E. Dopamine receptors and L-dopa-induced dyskinesia. Parkinsonism Relat Disord. 2009;15 suppl 4:S8-S12.

33. Cenci MA, Lee CS, Bjorklund A. L-DOPAinduced dyskinesia in the rat is associated with striatal overexpression of prodynorphin- and glutamic acid decarboxylase mRNA. Eur J Neurosci. 1998;10(8):2694-2706.

34. Lundblad M, Andersson M, Winkler C, Kirik D, Wierup N, Cenci MA. Pharmacological validation of behavioural measures of akinesia and dyskinesia in a rat model of Parkinson's disease. Eur J Neurosci. 2002;15(1):120-132.

35. Lois C, Hong EJ, Pease S, Brown EJ, Baltimore D. Germline transmission and tissue-specific expression of transgenes delivered by lentiviral vectors. Science. 2002;295(5556):868-872.

36. Schluter OM, Xu W, Malenka RC. Alternative $\mathrm{N}$-terminal domains of PSD-95 and SAP97 govern activity-dependent regulation of synaptic AMPA receptor function. Neuron. 2006;51(1):99-111.

37. Fasano S, et al. Inhibition of Ras-guanine nucleotide-releasing factor 1 (Ras-GRF1) signaling in the striatum reverts motor symptoms associated with L-dopa-induced dyskinesia. Proc Natl Acad SciU S A. 2010;107(50):21824-21829.

38. Vila M, et al. Consequences of nigrostriatal denervation on the functioning of the basal ganglia in human and nonhuman primates: an in situ hybridization study of cytochrome oxidase subunit I mRNA. J Neurosci. 1997;17(2):765-773.

39. Vila $M$, et al. Evolution of changes in neuronal activity in the subthalamic nucleus of rats with unilateral lesion of the substantia nigra assessed by metabolic and electrophysiological measurements. Eur J Neurosci. 2000;12(1):337-344

40. Boraud T, Bezard E, Bioulac B, Gross CE. Dopa- mine agonist-induced dyskinesias are correlated to both firing pattern and frequency alterations of pallidal neurones in the MPTP-treated monkey. Brain. 2001;124(pt 3):546-557.

41. Meissner W, et al. Increased slow oscillatory activity in substantia nigra pars reticulata triggers abnormal involuntary movements in the 6-OHDA-lesioned rat in the presence of excessive extracellular striatal dopamine. Neurobiol Dis. 2006; 22(3):586-598

42. Zhang $J$, et al. Inhibition of the dopamine D1 receptor signaling by PSD-95. J Biol Chem. 2007;282(21):15778-15789.

43. Goetz CG, Poewe W, Rascol O, Sampaio C. Evidence-based medical review update: pharmacological and surgical treatments of Parkinson's disease: 2001 to 2004. Mov Disord. 2005;20(5):523-539.

44. Rylander D, et al. A mGluR5 antagonist under clinical development improves L-DOPA-induced dyskinesia in parkinsonian rats and monkeys. Newrobiol Dis. 2010;39(3):352-361.

45. Carlsson A, Waters N, Holm-Waters S, Tedroff J, Nilsson M, Carlsson ML. Interactions between monoamines, glutamate, and GABA in schizophrenia: New Evidence. Annu Rev Pharmacol Toxicol. 2001;41:237-260.

46. Kennedy MB. Signal-processing machines at the postsynaptic density. Science. 2000;290(5492):750-754.

47. Kim E, Sheng M. PDZ domain proteins of synapses. Nat Rev Neurosci. 2004;5(10):771-781.

48. Groc L, et al. Surface trafficking of neurotransmitter receptor: comparison between single-molecule/quantum dot strategies. J Neurosci. 2007; 27(46):12433-12437.

49. Triller A, Choquet D. New concepts in synaptic biology derived from single-molecule imaging. Neuron. 2008;59(3):359-374.

50. Yao WD, et al. Identification of PSD-95 as a regulator of dopamine-mediated synaptic and behavioral plasticity. Neuron. 2004;41(4):625-638.

51. Bezard E, Gross CE, Qin L, Gurevich VV, Benovic JL, Gurevich EV. L-DOPA reverses the MPTPinduced elevation of the arrestin 2 and GRK6 expression and enhanced ERK activation in monkey brain. Neurobiol Dis. 2005;18(2):323-335.

52. Premont RT, Gainetdinov RR. Physiological roles of g protein-coupled receptor kinases and arrestins. Annu Rev Physiol. 2007;69:511-534.

53. Bernard V, Decossas M, Liste I, Bloch B. Intraneuronal trafficking of G-protein-coupled receptors in vivo. Trends Neurosci. 2006;29(3):140-147.

54. Gardoni $\mathrm{F}$, et al. A critical interaction between NR2B and MAGUK in L-DOPA induced dyskinesia. J Neurosci. 2006;26(11):2914-2922.

55. Paille V, et al. Distinct levels of dopamine denervation differentially alter striatal synaptic plasticity and NMDA receptor subunit composition. J Neurosci. 2010;30(42):14182-14193.

56. Tardin C, Cognet L, Bats C, Lounis B, Choquet D. Direct imaging of lateral movements of AMPA receptors inside synapses. EMBO J. 2003;22(18):4656-4665.

57. Ashby MC, Maier SR, Nishimune A, Henley JM. Lateral diffusion drives constitutive exchange of AMPA receptors at dendritic spines and is regulated by spine morphology. J Neurosci. 2006; 26(26):7046-7055.

58. Borgdorff AJ, Choquet D. Regulation of AMPA receptor lateral movements. Nature. 2002; 417(6889):649-653.

59. Groc L, et al. Differential activity-dependent regulation of the lateral mobilities of AMPA and NMDA receptors. Nat Neurosci. 2004;7(7):695-696.

60. Groc L, et al. NMDA receptor surface mobility depends on NR2A-2B subunits. Proc Natl Acad Sci US A. 2006;103(49):18769-18774.

61. Dahan M, Levi S, Luccardini C, Rostaing P, Riveau B, Triller A. Diffusion dynamics of glycine recep- 
tors revealed by single-quantum dot tracking. Science. 2003;302(5644):442-445.

62. Meier J, Vannier C, Serge A, Triller A, Choquet D. Fast and reversible trapping of surface glycine receptors by gephyrin. Nat Neurosci. 2001;4(3):253-260.

63. Bouzigues C, Dahan M. Transient directed motions of $\mathrm{GABA}(\mathrm{A})$ receptors in growth cones detected by a speed correlation index. Biophys J. 2007; 92(2):654-660.

64. Hegener O, Prenner L, Runkel F, Baader SL, Kappler J, Haberlein H. Dynamics of beta2-adrenergic receptor-ligand complexes on living cells. Biochemistry. 2004;43(20):6190-6199.

65. Lober RM, Pereira MA, Lambert NA. Rapid activation of inwardly rectifying potassium channels by immobile G-protein-coupled receptors. J Neurosci. 2006;26(48):12602-12608.

66. Scott L, et al. Allosteric changes of the NMDA receptor trap diffusible dopamine 1 receptors in spines. Proc Natl Acad Sci U S A. 2006;103(3):762-767.

67. Lee FJ, et al. Dual regulation of NMDA receptor functions by direct protein-protein interactions with the dopamine D1 receptor. Cell. 2002;111(2):219-230.

68. Fiorentini C, et al. Loss of synaptic D1 dopamine/N-methyl-D-aspartate glutamate receptor complexes in L-DOPA-induced dyskinesia in the rat. Mol Pharmacol. 2006;69(3):805-812.

69. Verhagen Metman L, Del Dotto P, van den Munckhof P, Fang J, Mouradian MM, Chase TN. Amantadine as treatment for dyskinesias and motor fluctuations in Parkinson's disease. Neurology. 1998;50(5):1323-1326.

70. Sawada $\mathrm{H}$, et al. Amantadine for dyskinesias in Parkinson's disease: a randomized controlled trial. PLoS One. 2010;5(12):e15298.

71. Schwarze SR, Ho A, Vocero-Akbani A, Dowdy SF. In vivo protein transduction: delivery of a biologically active protein into the mouse. Science. 1999;285(5433):1569-1572.

72. Jo YH, Donier E, Martinez A, Garret M, Toulme E, Boue-Grabot E. Cross-talk between P2X4 and gamma-aminobutyric acid, type A receptors determines synaptic efficacy at a central synapse. J Biol Chem. 2011;286(22):19993-20004.

73. Schuster S, et al. The 3-hydroxy-3-methylglutarylCoA reductase inhibitor lovastatin reduces severity of L-DOPA-induced abnormal involuntary movements in experimental Parkinson's disease. J Neurosci. 2008;28(17):4311-4316.

74. Torres GE, Gainetdinov RR, Caron MG. Plasma membrane monoamine transporters: structure, regulation and function. Nat Rev Neurosci. 2003;4(1):13-25.

75. Olsson M, Nikkhah G, Bentlage C, Bjorklund A Forelimb akinesia in the rat Parkinson model: differential effects of dopamine agonists and nigral transplants as assessed by a new stepping test. J Neurosci. 1995;15(5 pt 2):3863-3875.

76. Pioli EY, Meissner W, Sohr R, Gross CE, Bezard E, Bioulac BH. Differential behavioral effects of partial bilateral lesions of ventral tegmental area or substantia nigra pars compacta in rats. Neuroscience. 2008;153(4):1213-1224.

77. Winkler C, Kirik D, Bjorklund A, Cenci MA. L-DOPA-induced dyskinesia in the intrastriatal 6-hydroxydopamine model of parkinson's disease: relation to motor and cellular parameters of nigrostriatal function. Neurobiol Dis. 2002;10(2):165-186.

78. Bezard E, et al. Relationship between the appearance of symptoms and the level of nigrostriatal degeneration in a progressive 1-methyl-4-phenyl1,2,3,6-tetrahydropyridine-lesioned macaque model of Parkinson's disease. J Neurosci. 2001; 21(17):6853-6861.

79. Guigoni C, et al. Pathogenesis of levodopa-induced dyskinesia: focus on D1 and D3 dopamine receptors. Parkinsonism Relat Disord. 2005;11(suppl 1):S25-S29.

80. Schuster S, et al. Antagonizing L-type Ca2+ channel reduces development of abnormal involuntary movement in the rat model of L-3,4-dihydroxyphenylalanine-induced dyskinesia. Biol Psychiatry. 2009;65(6):518-526.

81. Pfaffl MW, Horgan GW, Dempfle L. Relative expression software tool (REST) for group-wise comparison and statistical analysis of relative expression results in real-time PCR. Nucleic Acids Res. 2002;30(9):e36.

82. Bezard E, et al. Attenuation of levodopa-induced dyskinesia by normalizing dopamine D3 receptor function. Nat Med. 2003;9(6):762-767.

83. Bezard E, et al. Relationship between the appearance of symptoms and the level of nigrostriatal degeneration in a progressive MPTP-lesioned macaque model of Parkinson's disease. J Neurosci. 2001;21(17):6853-6861.

84. Fernagut PO, et al. Dopamine transporter binding is unaffected by L-DOPA administration in normal and MPTP-treated monkeys. PLoS One. 2010;5(11):e14053.

85. Gold SJ, et al. RGS9-2 negatively modulates L-3,4-dihydroxyphenylalanine-induced dyskinesia in experimental Parkinson's disease. J Neurosci. 2007;27(52):14338-14348

86. Caille I, Dumartin B, Le Moine C, Begueret J, Bloch B. Ontogeny of the D1 dopamine receptor in the rat striatonigral system: an immunohistochemical study. EurJ Neurosci. 1995;7(4):714-722.

87. Kowalski C, Giraud P. Dopamine decreases striatal enkephalin turnover and proenkephalin messenger RNA abundance via D2 receptor activation in primary striatal cell cultures. Neuroscience. 1993;53(3):665-672.

88. Martin-Negrier M, Charron G, Bloch B. Agonist stimulation provokes dendritic and axonal dopamine $\mathrm{D}(1)$ receptor redistribution in primary cultures of striatal neurons. Neuroscience. 2000;99(2):257-266.

89. Martin-Negrier ML, Charron G, Bloch B. Receptor recycling mediates plasma membrane recovery of dopamine D1 receptors in dendrites and axons after agonist-induced endocytosis in primary cultures of striatal neurons. Synapse. 2006;60(3):194-204.

90. Bats C, Groc L, Choquet D. The interaction between Stargazin and PSD-95 regulates AMPA receptor surface trafficking. Neuron. 2007;53(5):719-734.

91. Heine M, et al. Surface mobility of postsynaptic AMPARs tunes synaptic transmission. Science. 2008;320(5873):201-205. 